\title{
Search for the associated production of a Higgs boson with a single top quark in proton-proton collisions at $\sqrt{s}=8 \mathrm{TeV}$
}

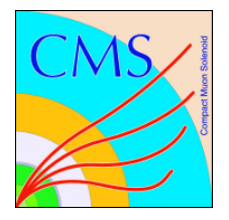

\section{The CMS collaboration}

E-mail: cms-publication-committee-chair@cern.ch

ABSTRACT: This paper presents the search for the production of a Higgs boson in association with a single top quark ( $\mathrm{tHq})$, using data collected in proton-proton collisions at a center-of-mass energy of $8 \mathrm{TeV}$ corresponding to an integrated luminosity of $19.7 \mathrm{fb}^{-1}$. The search exploits a variety of Higgs boson decay modes resulting in final states with photons, bottom quarks, and multiple charged leptons, including tau leptons, and employs a variety of multivariate techniques to maximize sensitivity to the signal. The analysis is optimized for the opposite sign of the Yukawa coupling to that in the standard model, corresponding to a large enhancement of the signal cross section. In the absence of an excess of candidate signal events over the background predictions, 95\% confidence level observed (expected) upper limits on anomalous tHq production are set, ranging between 600 (450) fb and 1000 (700) fb depending on the assumed diphoton branching fraction of the Higgs boson. This is the first time that results on anomalous tHq production have been reported.

KEYwords: Hadron-Hadron scattering (experiments), Higgs physics, Top physics

ArXiv EPrint: 1509.08159 


\section{Contents}

1 Introduction 1

2 The CMS detector, event reconstruction, and simulation 3

3 Description of the analyses 5

$3.1 \mathrm{H} \rightarrow \gamma \gamma$ channel

$3.2 \mathrm{H} \rightarrow \mathrm{b} \overline{\mathrm{b}}$ channel 8

3.2.1 Event selection $\quad 8$

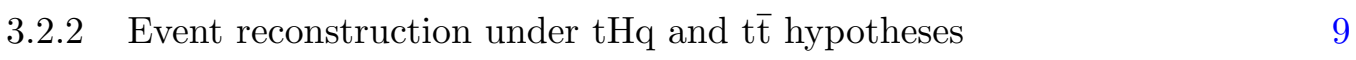

$\begin{array}{ll}3.2 .3 \quad \text { Event classification and signal extraction } & 10\end{array}$

$\begin{array}{lll}3.3 \mathrm{H} \rightarrow \text { WW channel } & 10\end{array}$

$\begin{array}{lll}\text { 3.3.1 Background modeling } & 11\end{array}$

$\begin{array}{lll}\text { 3.3.2 Event selection and signal extraction } & 13\end{array}$

$\begin{array}{lll}3.4 \mathrm{H} \rightarrow \tau^{+} \tau^{-} \text {channel } & 14\end{array}$

$\begin{array}{lll}3.4 .1 & \text { Event selection } & 15\end{array}$

$\begin{array}{ll}3.4 .2 \text { Background modeling } & 15\end{array}$

$\begin{array}{lll}3.4 .3 & \text { Signal extraction } & 16\end{array}$

$\begin{array}{lll}4 & \text { Systematic uncertainties } & 18\end{array}$

$\begin{array}{llr}5 & \text { Results } & 20\end{array}$

$\begin{array}{lll}6 & \text { Summary } & 21\end{array}$

A Selected distributions of inputs to the multivariate discriminants $\quad 23$

$\begin{array}{lr}\text { The CMS collaboration } & 29\end{array}$

\section{Introduction}

The discovery of a Higgs boson by the ATLAS and CMS experiments in 2012 [1-3] opened a new field for exploration in particle physics. The Higgs boson was discovered through its direct coupling to other known heavy bosons (W, Z) and its indirect coupling to photons, which in the standard model (SM) occurs via a loop involving $\mathrm{W}$ bosons or top quarks. Strong evidence for the Higgs boson coupling to fermions has also been established [4, 5]. Moreover, there is evidence of the Higgs boson coupling to bottom quarks from the Tevatron [6] and from CMS [7], and to tau leptons from ATLAS [5] and CMS [8]. It is now critical to test whether the observed Higgs boson is the SM Higgs boson by studying its coupling to other elementary particles. 

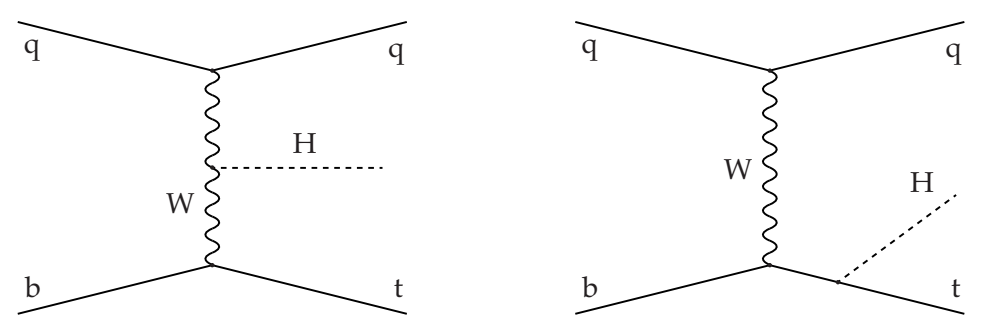

Figure 1. Dominant Feynman diagrams for the production of tHq events: the Higgs boson is typically radiated from the heavier particles of the diagram, i.e. the $\mathrm{W}$ boson (left) or the top quark (right).

The coupling of the new boson to the top quark is of special interest. Because of its very large mass [9] the top quark is widely believed to play a special role in the mechanism of electroweak symmetry breaking. Physics beyond the SM could modify the top quark Yukawa coupling without violating current experimental constraints. The most straightforward way to study this coupling is through the measurement of top quark-antiquark pair production in association with a Higgs boson $(\mathrm{t} \overline{\mathrm{t}} \mathrm{H})$, as was recently done by ATLAS and CMS [10-13]. Interactions of the Higgs boson with the top quark can also be probed by studying the associated production of a single top quark and a Higgs boson, which proceeds mainly through $t$-channel diagrams (tHq) [14] in which the Higgs boson is emitted either from an internally exchanged $\mathrm{W}$ boson or from a top quark, as shown in figure 1. The associated single top quark and Higgs boson production can also be accompanied by a $\mathrm{W}$ in the final state (tHW). As the couplings of the Higgs boson to the $\mathrm{W}$ boson and the top quark have opposite signs in the SM, these two diagrams interfere destructively. The cross section for single top quark plus Higgs boson production via the tHq process in pp collisions at a center-of-mass energy of $8 \mathrm{TeV}$ has been calculated to be about $18 \mathrm{fb}$ at next-to-leading-order (NLO) [15].

Anomalous coupling of the Higgs boson to SM particles would modify the expected rate of tHq events [16]. A number of models have been proposed that would modify the interference between the diagrams involving t $\bar{t} H$ and WWH couplings. For example, a negative coupling of the Higgs boson to the top quark $\left(C_{\mathrm{t}}=-1\right)$ would give rise to about a 15-fold increase in the tHq cross section. Recent work suggests the investigation of anomalous tHq production in events with a pair of photons [17, 18], b quarks [15], or multiple leptons in the final state [18]. The same interference probes the CP-violating phase of the top quark Yukawa coupling [19-21]. Also, a large rate of single top quark plus Higgs boson events could signal the direct production of heavy new particles as predicted in composite and little Higgs models [22], or new physics showing up as Higgs boson mediated flavor changing neutral currents [23]. The apparent exclusion of the $C_{\mathrm{t}}=-1$ case based on the value of the branching fraction for $\mathrm{H} \rightarrow \gamma \gamma$ only holds under the assumption that no new particles contribute to the loop in the main diagram for that decay [24].

This paper reports the first search for tHq production, focusing on the scenario where the coupling of the Higgs boson to the top quark has a sign opposite to that predicted 
by the SM, using data collected by the CMS experiment at the CERN LHC. Four Higgs boson decay modes are explored. Section 2 describes the CMS detector, the reconstruction algorithms, and the simulated samples. Section 3 outlines the selection, background modeling, and signal extraction techniques for analyses based on $\mathrm{H}$ decay channels with photons, hadrons, and multiple leptons. Section 4 describes the systematic uncertainties affecting the search results. Finally, the procedure for combining the results of the searches is presented in section 5 . The results are summarized in section 6 .

\section{The CMS detector, event reconstruction, and simulation}

The central feature of the CMS apparatus is a superconducting solenoid of $6 \mathrm{~m}$ internal diameter, providing an axial magnetic field of $3.8 \mathrm{~T}$. Within the magnet volume, there are a silicon pixel and strip tracker, a lead tungstate crystal electromagnetic calorimeter (ECAL), and a brass and scintillator hadron calorimeter (HCAL), each composed of a barrel and two endcap sections. The tracking detectors provide coverage for charged particles within pseudorapidity $|\eta|<2.5$. The ECAL and HCAL calorimeters provide coverage up to $|\eta|<3.0$. The ECAL is divided into two distinct regions: the barrel region, which covers $|\eta|<1.48$, and the endcap region, which covers $1.48<|\eta|<3.00$. A quartz-fiber forward calorimeter extends the coverage further up to $|\eta|<5.0$. Muons are measured in gas-ionization detectors embedded in the steel flux-return yoke outside the solenoid. A detailed description of the CMS detector, together with a definition of the coordinate system used and the relevant kinematic variables, can be found in ref. [25].

The particle-flow (PF) event reconstruction algorithm $[26,27]$ consists of reconstructing and identifying each single particle with an optimized combination of all subdetector information. The energy of charged hadrons is determined from a combination of their momentum measured in the tracker and the matching ECAL and HCAL energy deposits.

Photon PF candidates are reconstructed from the energy deposits in the ECAL, grouping the individual clusters into a supercluster. The superclustering algorithms achieve an almost complete reconstruction of the energy of photons (and electrons) that convert into electron-positron pairs (emit bremsstrahlung) in the material in front of the ECAL. The photon candidates are identified within the ECAL fiducial region $|\eta|<2.5$, excluding the barrel-endcap transition region $1.44<|\eta|<1.57$, where photon reconstruction is suboptimal. Isolation requirements are applied to photon candidates by looking at neighboring particle candidates. In the barrel section of the ECAL, an energy resolution of about $1 \%$ is achieved for unconverted or late-converting photons in the tens of $\mathrm{GeV}$ energy range. The remaining barrel photons have a resolution of about $1.3 \%$ up to a pseudorapidity of $|\eta|=1$, rising to about $2.5 \%$ at $|\eta|=1.4$. In the endcaps, the resolution of unconverted or late-converting photons is about $2.5 \%$, while the remaining endcap photons have a resolution between 3 and $4 \%$. Additional details on photon reconstruction and identification can be found in refs. [28, 29].

Electrons with $p_{\mathrm{T}}$ greater than $7 \mathrm{GeV}$ are reconstructed within the geometrical acceptance of the tracker, $|\eta|<2.5$. The electron momentum is determined from the combination of ECAL and tracker measurements. Electron identification relies on a multivariate (MVA) 
technique, which combines observables sensitive to the amount of bremsstrahlung along the electron trajectory, the spatial and momentum matching between the electron trajectory and associated clusters, and shower shape observables [29, 30]. In order to increase the lepton efficiency, the $\mathrm{H} \rightarrow$ leptons analysis uses a looser selection for the MVA discriminant than do the other analysis channels.

Muons with $p_{\mathrm{T}}>5 \mathrm{GeV}$ are reconstructed within $|\eta|<2.4$ [31]. The reconstruction combines information from both the silicon tracker and the muon spectrometer. The PF muons are selected from the reconstructed muon track candidates by applying minimal requirements on the track components in the muon and tracker systems and taking into account matching with energy deposits in the calorimeters [32].

Particles reconstructed with the $\mathrm{PF}$ algorithm are clustered into jets using the anti- $k_{\mathrm{T}}$ algorithm with a distance parameter of $0.5[33,34]$. Jet energy corrections are applied to account for the non-linear response of the calorimeters to the particle energies and other detector effects. These include corrections due to additional interactions within a beam crossing (pileup), where the average energy density from the extra interactions is evaluated on an event-by-event basis and the corresponding energy is subtracted from each jet [35]. The jet energy resolution is also modified in simulation with a smearing technique to match what is measured in data [36]. In all the final states that are studied, jets with $|\eta|<5.0$ and transverse momentum down to $20 \mathrm{GeV}$ are considered, though the final selection depends on the specific analysis.

The hadronic decay of a $\tau$ lepton $\left(\tau_{\mathrm{h}}\right)$ produces a narrow jet of charged and neutral hadrons, which are mostly pions. Each neutral pion subsequently decays into a pair of photons. The identification of $\tau_{\mathrm{h}}$ jets begins with the formation of $\mathrm{PF}$ jets by clustering charged hadron and photon objects via the anti- $k_{\mathrm{T}}$ algorithm. Additional details on $\tau$ reconstruction and identification can be found in ref. [37]. For this analysis, decays involving one or three charged hadrons are used.

The missing transverse momentum vector $\vec{p}_{\mathrm{T}}^{\text {miss }}$ is defined as the negative projection on the plane perpendicular to the beams of the vectorial sum of the momenta of all reconstructed PF candidates in an event. Its magnitude is referred to as $E_{\mathrm{T}}^{\mathrm{miss}}$.

Jets are identified as originating from $b$ quark production (b tagged) using an algorithm based on the combined properties of secondary vertices and track-based lifetime information, known as the combined secondary vertex (CSV) tagging algorithm [38, 39]. Different working points are chosen for the various analyses: a loose working point providing an efficiency for b quark jets of about $85 \%$ and a light-flavor jet misidentification (mistag) rate of $10 \%$, a medium working point with $70 \%$ b-quark jet efficiency and $1 \%$ light-flavor jet mistag rate, and a tight working point with $50 \%$ b-quark jet efficiency and $0.1 \%$ lightflavor jet mistag rate. Only jets with $|\eta|<2.4$ (within the CMS tracker acceptance) are identified with this technique.

A number of Monte Carlo (MC) event generators are used to simulate the signal and backgrounds. Signal events are produced with MADGRAPH (v5.1.3.30) [40], with a non-SM Yukawa coupling of $C_{\mathrm{t}}=-1$, and then passed through PYтнIA (v6.426) [41] to add an underlying event and to perform parton showering and hadronization. The masses for the top quark and Higgs boson are set to 173 and $125 \mathrm{GeV}$, respectively. The CTEQ6L1 [42] 
parton distribution function (PDF) set is used. The sample is produced either using the five-flavor scheme or the four-flavor scheme. Processes such as t $\bar{t}$ plus additional particles (heavy-flavor jets, light-flavor jets, gluons, or bosons), W/Z plus jets, and di- and tri-boson production are all generated with MADGRAPH. Single top quark plus jets and inclusive Higgs boson production are generated with POWHEG (v1.0, r1380) [43, 44]. Both multijet

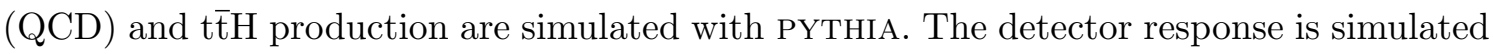
using a detailed description of the CMS detector based on the GEANT4 package [45]. All processes have been normalized to the most recent theoretical cross section computations.

The simulated samples are reweighted to represent the pileup distribution as measured in the data. To match the performance of reconstructed objects between data and simulation, the latter is corrected with a set of data/MC scale factors. Leptons are corrected for the difference in trigger efficiency, as well as in lepton identification and isolation efficiency. Corrections accounting for residual differences between data and simulation are applied to the ECAL energy before combining the energy with the momentum determined from the tracker for electrons. Similar corrections are applied to the muon momentum.

\section{Description of the analyses}

The $t$-channel single top quark plus Higgs boson process has, at tree level, three particles in the final state: a top quark, a Higgs boson, and an additional quark jet, which tends to be emitted in the forward region. A spectator $b$ quark is produced through splitting of a gluon in the incoming proton, resulting in an additional bottom-flavor jet (b jet) that can enter the detector acceptance. Other Higgs boson production mechanisms, such as $\mathrm{t} \overline{\mathrm{t}} \mathrm{H}$, are considered as background. All of the analyses make use of the leptonic decay of the top quark, which yields a high-momentum lepton and an identifiable b jet. Requiring these objects in the event improves the signal-to-background ratio for each analysis. The analyses are distinguished by the Higgs boson decay channel, as described in the following subsections.

\section{$3.1 \quad \mathrm{H} \rightarrow \gamma \gamma$ channel}

The diphoton branching fraction of the Higgs boson in the standard model is very small $(0.23 \%)$ but the diphoton final state allows very good background rejection thanks to the excellent diphoton invariant mass resolution provided by the CMS detector. A negative top quark Yukawa coupling would not only enhance the yield of tHq events, but also more than double the rate of Higgs bosons decaying to diphotons. Thus the diphoton final state of the Higgs boson decay in tHq events is expected to be highly sensitive to the top quark Yukawa coupling.

The data for the diphoton analysis are collected using diphoton triggers with two different photon identification schemes. One requires calorimetric identification based on the electromagnetic shower shape and isolation of the photon candidate. The other requires only that the photon has a high value of the R9 shower shape variable, which is defined as the ratio of the energy contained in a $3 \times 3$ array of ECAL crystals centered on the most energetic deposit in the supercluster to the energy of the whole supercluster. The 
$E_{\mathrm{T}}$ thresholds at trigger level are $26(18) \mathrm{GeV}$ and $36(22) \mathrm{GeV}$ on the leading (subleading) photon depending on the running period. To maintain a high signal efficiency, trigger paths based on both photon identification schemes are combined in the offline data selection.

The event selection requires the presence of two photons, with the transverse momentum of the leading photon $\left(p_{\mathrm{T} 1}\right)$ greater than $50 m_{\gamma \gamma} / 120$, where $m_{\gamma \gamma}$ is the reconstructed invariant mass of the diphoton system, and that of the subleading photon greater than $25 \mathrm{GeV}$. The stringent requirement on $p_{\mathrm{T} 1}$ is found to have very high efficiency (>98\%) for the signal and reduces the contributions of nonresonant backgrounds. The presence of exactly one isolated electron or muon with $p_{\mathrm{T}}>10 \mathrm{GeV}$ and at least one $\mathrm{b}$ quark jet with $p_{\mathrm{T}}>20 \mathrm{GeV}$ are required to identify the leptonic decay of the top quark. If more than one jet is b tagged, the one with the largest transverse momentum is chosen as the b jet candidate from the top quark decay. Finally, the highest $p_{\mathrm{T}}$ jet in the event that is not $\mathrm{b}$ tagged must have $p_{\mathrm{T}}>20 \mathrm{GeV}$ and $|\eta|>1$.

After applying these requirements, a multivariate method is used to further reduce the $\mathrm{t} \overline{\mathrm{t}} \mathrm{H}$ contribution. A Bayes classifier, L, is constructed as the ratio of signal over signal plus background likelihoods for a chosen set of discriminating observables:

$$
\mathrm{L}(\mathrm{x})=\frac{\mathrm{L}_{\mathrm{S}}(\mathrm{x})}{\mathrm{L}_{\mathrm{S}}(\mathrm{x})+\mathrm{L}_{\mathrm{B}}(\mathrm{x})}
$$

For each event the signal $\left(\mathrm{L}_{\mathrm{S}}\right)$ and background $\left(\mathrm{L}_{\mathrm{B}}\right)$ likelihoods are calculated as the product of the respective signal and background probability density functions $(p)$, evaluated at the observed values $\left(\mathrm{x}^{j}\right)$ :

$$
\mathrm{L}^{i}(\mathrm{x})=\prod_{j} p_{j}^{i}\left(\mathrm{x}^{j}\right)
$$

where $i$ stands for each signal or background process and $j$ for each variable considered. The classifier is built from the following variables: the jet multiplicity in the event; the transverse mass of the top quark using the lepton, the candidate $b$ jet and the missing transverse momenta; the pseudorapidity of the light quark candidate; the rapidity gap between the lepton and the forward jet; and the charge of the lepton candidate. The last observable is chosen as the pp initial state is more likely to produce a top quark rather than a top antiquark. All these variables are observed to discriminate well between simulated $\mathrm{t} \overline{\mathrm{t}} \mathrm{H}$ and tHq events [46]. The linear correlation coefficients for the input variables are all less than $10 \%$ for both signal and background processes. The classifier value is required to be greater than 0.25 , to suppress the $t \bar{t} H$ contribution to the signal sample. This requirement retains about $90 \%$ of the signal events.

The invariant mass of the diphoton system is the primary search variable for a signallike excess, as the signal would appear as a narrow diphoton resonance centered at the known Higgs boson mass $m_{\mathrm{H}}=125 \mathrm{GeV}$.

The backgrounds can be classified according to their resonant or nonresonant behavior in the diphoton system; a different approach has been adopted to estimate the rate from each category. Resonant backgrounds give rise to a Higgs boson decaying to two photons in the final state. These backgrounds are dominated by the $t \bar{t} \mathrm{H}$ process and also include Higgs 

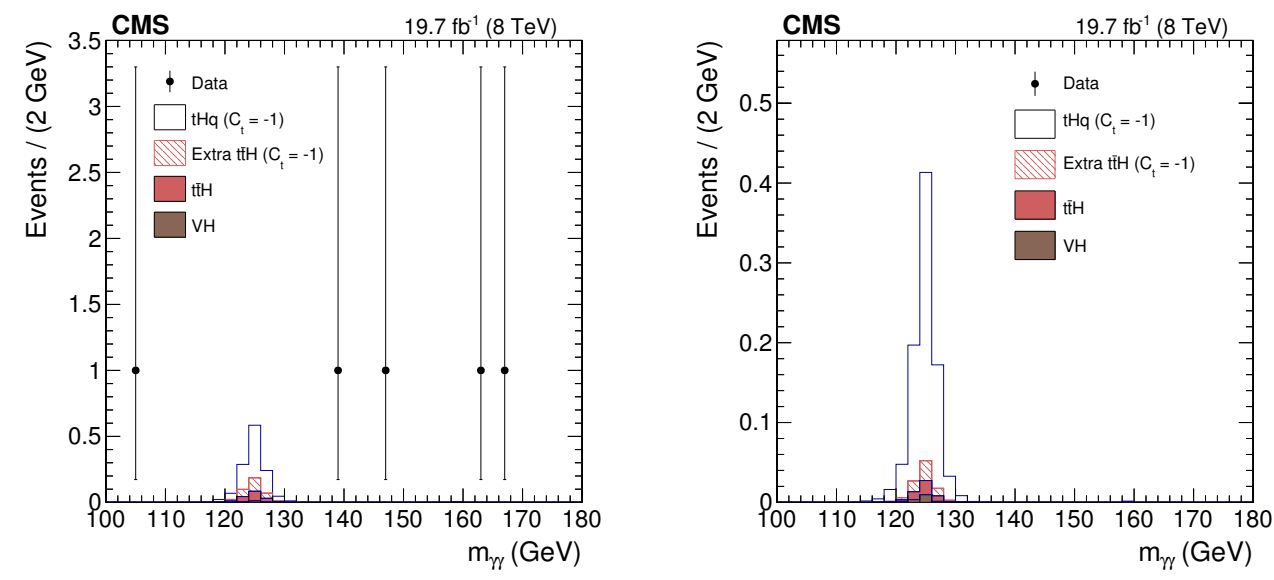

Figure 2. Invariant mass of the diphoton system for events passing the event selection requirements, but for the likelihood discriminant cut (left), and for events passing the full selection (right). The data (black markers) are compared to the MC simulation (stacked histograms). No events are observed after the requirement on the likelihood discriminant.

\begin{tabular}{|ll|}
\hline Process & Events \\
\hline $\mathrm{tHq}\left(C_{\mathrm{t}}=-1\right)$ & 0.67 \\
\hline $\mathrm{t} \overline{\mathrm{t}} \mathrm{V}$ & $0.03+0.05^{\dagger}$ \\
$\mathrm{VH}$ & $0.01+0.01^{\dagger}$ \\
Other $\mathrm{H}$ & 0 \\
\hline Data & 0 \\
\hline
\end{tabular}

Table 1. Expected yields for the diphoton analysis, based on simulations. Yields are counted for

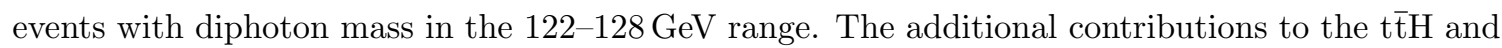
VH processes arising from the enhanced Higgs to diphoton branching fraction due to the $C_{\mathrm{t}}=-1$ assumption are marked with a dagger $\left(^{\dagger}\right)$.

production in association with a vector boson ( $\mathrm{VH}$ ); they appear as an additional contribution under the expected signal peak, and are evaluated using MC simulation. Nonresonant backgrounds are evaluated from the $m_{\gamma \gamma}$ sidebands. The main nonresonant background processes include diphoton production in association with jets $(\gamma \gamma+$ jets $)$, single-photon production in association with jets $(\gamma+$ jets $)$, and diphoton events produced in association with top quarks $(\mathrm{t} \overline{\mathrm{t}} \gamma \gamma, \mathrm{t} \gamma \gamma)$.

The signal region is defined as the $\pm 3 \mathrm{GeV}$ range around the nominal Higgs boson mass. While the contribution of resonant backgrounds is taken from the simulation, nonresonant backgrounds are evaluated by counting the events in the $m_{\gamma \gamma}$ sidebands $100 \mathrm{GeV}<m_{\gamma \gamma}<\left(m_{\mathrm{H}}-3 \mathrm{GeV}\right)$ and $\left(m_{\mathrm{H}}+3 \mathrm{GeV}\right)<m_{\gamma \gamma}<180 \mathrm{GeV}$, which have negligible signal contamination.

The event yields in the signal region are shown in table 1 . The selection has an expected efficiency of $17 \%$ for tHq events in the diphoton decay channel. Figure 2 shows the $m_{\gamma \gamma}$ spectrum for events passing the event selection before and after the likelihood requirement. 
No events pass the selection. In order to model the nonresonant background shape using data, a control region with relaxed $\mathrm{b}$ tagging requirements is defined. The functional form chosen for the $m_{\gamma \gamma}$ distribution of background events is an exponential, and the uncertainty in the knowledge of the background shape is assessed by defining an orthogonal control region in which the isolation requirements on one of the two photons are inverted. This uncertainty amounts to $33 \%$. The number of events observed and the systematic uncertainties are later used to set a limit on the rate of tHq production.

\section{2 $\mathrm{H} \rightarrow \mathrm{b} \overline{\mathrm{b}}$ channel}

The search for $\mathrm{tHq}$ in the $\mathrm{H} \rightarrow \mathrm{b} \overline{\mathrm{b}}$ decay final state benefits from the large Higgs to bottom-antibottom quarks branching fraction, but suffers from significant backgrounds from tét events.

\subsubsection{Event selection}

The analysis is performed with data collected with two triggers: one requiring an electron candidate with $p_{\mathrm{T}}>27 \mathrm{GeV}$ and $|\eta|<2.4$, the other requiring a muon candidate with $p_{\mathrm{T}}>24 \mathrm{GeV}$ and $|\eta|<2.1$. In each case the lepton must be isolated. The effect of the triggers is emulated in all simulated data sets. An event in the electron (muon) channel is required to contain exactly one electron (muon) candidate with $p_{\mathrm{T}}>30(26) \mathrm{GeV}$ and pass a set of identification criteria labeled as "tight". In order to reject Drell-Yan (DY) and other processes with multiple prompt leptons, events are rejected if additional leptons exist that pass a looser criterion.

The signal final state in this channel is expected to contain at least five quarks: two b quarks from the Higgs boson decay, one b quark each from the top quark decay and from the strong interaction, and a forward light quark from the $t$-channel process. Each event is thus required to contain at least four jets with $p_{\mathrm{T}}>30 \mathrm{GeV}$ and the threshold for counting additional jets beyond the fourth is chosen to be $20 \mathrm{GeV}$. Jets with $|\eta|>2.4$ are considered only if they have $p_{\mathrm{T}}>40 \mathrm{GeV}$. A tight working point of the CSV b tagging algorithm is chosen to suppress the large background from top quark pair production, which contains a smaller number of genuine $\mathrm{b}$ quarks than the signal process. This working point has typical tagging efficiencies of $55 \%$ for $\mathrm{b}$ jets and $0.1 \%$ for light-flavor jets. To reject multijet events, a missing transverse energy selection is applied with thresholds optimized

per channel: $E_{\mathrm{T}}^{\text {miss }}>45 \mathrm{GeV}$ in the electron channel and $E_{\mathrm{T}}^{\text {miss }}>35 \mathrm{GeV}$ in the muon channel. As the b quark produced in the strong interaction of the tHq process is often forward and falls outside the acceptance of the detector, two analysis samples are defined: one of events containing at least four jets with three of them b-tagged and one of events containing at least five jets with four of them b-tagged. Additionally, a two-tag control sample dominated by t $\bar{t}$ plus jets events is used for validation of event reconstruction and signal extraction techniques described in the following section.

After this event selection is applied, the sample is dominated by the t $\overline{\mathrm{t}}$ plus jets background as well as other background contributions [47]. The three-tag sample has an expected signal-to-background ratio of $0.7 \%$. The four-tag sample has an improved ratio of approximately $2 \%$ but suffers from a limited number of events. The background kinematic 
distributions and normalizations are taken from simulation and are adjusted in the final fit, taking into account all systematic uncertainties, which are described in more detail in section 4. A cross-check approach that uses control data samples to model the dominant $t \bar{t}$ plus jets background in the signal regions by employing b-tagging and mistagging efficiencies in the two-tag control sample gives consistent results.

\subsubsection{Event reconstruction under tHq and $t \overline{\mathrm{t}}$ hypotheses}

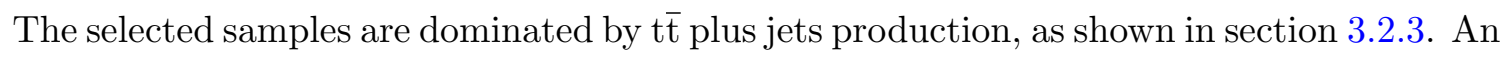
artificial neural network $(\mathrm{NN})$ is employed to separate the signal process from background, based on the features of tHq and t $\bar{t}$ plus jets events. Prior to this, a correspondence between reconstructed jets and the final-state objects must be built in order to define the input variables to the NN. For this purpose, each event is reconstructed under two hypotheses:

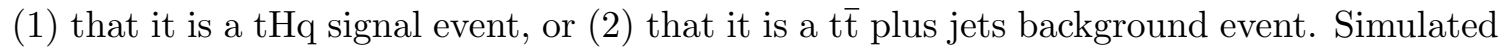
events are used to assess the correctness of the assignment of jets to quarks.

For the jet assignment under the tHq hypothesis in a simulated tHq event, all possible ways to assign four reconstructed jets to the four final state quarks from tHq $\rightarrow 3 \mathrm{bq} \ell \nu$ are considered, where a correct event interpretation is present in the case where four jets can be matched to the appropriate quarks within a cone of radius $\Delta R=\sqrt{(\Delta \eta)^{2}+(\Delta \phi)^{2}}=0.3$. If the distance between at least one quark and its assigned jet is larger than this threshold, the event interpretation is flagged as wrong. The total number of possible interpretations is reduced by additional requirements: because of $\mathrm{b}$ tagging considerations, $\mathrm{b}$ quarks can only be associated with central jets $(|\eta|<2.4)$, while only a jet failing the $\mathrm{b}$ tagging requirement can be assigned to the light recoil quark.

A NN is trained on tHq events to distinguish between correct and wrong interpretations with variables employing kinematic characteristics of the signal, like the $p_{\mathrm{T}}$ of the softest jet from the Higgs boson decay, the $|\eta|$ of the recoil jet, and the $\Delta R$ between the reconstructed top quark and the Higgs boson. Other variables include information such as b tagging or the reconstructed jet charge. The interpretation chosen for use in the analysis is the one that gives the largest NN response from all possible tHq jet assignments.

Similarly, another NN is used for the interpretation of events under the assumption that they originate from semileptonic $t \overline{\mathrm{t}}$ decays. The $\mathrm{NN}$ is trained with $\mathrm{t} \overline{\mathrm{t}} \rightarrow 2 \mathrm{~b} 2 \mathrm{q} \ell \nu$ simulated events, using both correct and wrong quark jet assignments in analogy with the tHq jet assignment described above. The number of possible jet-quark combinations is restricted by requiring that only b-tagged jets can be assigned to the two b quarks. The set of variables used under a $t \bar{t}$ event interpretation is similar to the one of the tHq event interpretation. It makes use of kinematic relations between objects, such as the $\Delta R$ between the $\mathrm{b}$ and $\mathrm{W}$ boson from the hadronically decaying top quark, or the difference between the reconstructed top quark mass and $\mathrm{W}$ boson mass in the hadronic top quark decay. It also employs $\mathrm{b}$ tagging information and relations between the jet and lepton charges. The jet assignment yielding the largest NN response is chosen as the event interpretation under the t $\bar{t}$ hypothesis. Additional details regarding the event interpretation can be found in ref. [47]. 


\subsubsection{Event classification and signal extraction}

The $\mathrm{tHq}$ and $\mathrm{t} \overline{\mathrm{t}}$ plus jets reconstruction algorithms described above are carried out on every event passing the selection criteria. This allows the construction of two sets of observables, where one set describes the event under the tHq hypothesis and the other the event under

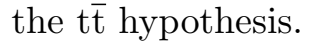

These two sets, together with the lepton charge, form the list of input variables for the final NN, which classifies events as signal- or background-like: $|\eta|$ of the recoil jet; number of b-tagged jets among the two jets from the Higgs boson decay; $p_{\mathrm{T}}$ of the Higgs boson; $p_{\mathrm{T}}$ of the recoil jet; $\Delta R$ between the two light-flavor jets from the hadronic top quark decay; reconstructed mass of the hadronically decaying top quark; number of b-tagged jets among the two light-flavor jets from the hadronic decay of the top quark; and lepton charge [47].

Figure 3 shows the distributions of the final event classifier in the three-tag and fourtag samples, separated by lepton flavor. The distributions of the NN outputs are used to extract the signal and to derive the upper limit on the cross section for tHq production. The normalizations of the distributions are taken from the result of a maximum likelihood fit where each background and the signal process are allowed to float within the assigned systematic and statistical uncertainties. The resulting distributions show a good agreement with data and residual differences are well covered by the total uncertainties.

\section{$3.3 \quad \mathrm{H} \rightarrow \mathrm{WW}$ channel}

The Higgs boson decay to two $\mathrm{W}$ bosons (with one boson off-shell) has the second-largest branching fraction in the standard model. The associated $\mathrm{tHq}, \mathrm{H} \rightarrow \mathrm{WW}$ and $\mathrm{t} \rightarrow \mathrm{Wb}$ final state allows several combinations of leptonically and/or hadronically decaying $\mathrm{W}$ bosons. Two channels are exploited here, in which either all three $\mathrm{W}$ bosons decay leptonically, or the pair of $\mathrm{W}$ bosons with equal charge, resulting in a signature of either three leptons (electrons or muons), or two same-sign leptons with two light quark jets. The tHW process can also result in this set of leptons. In the tHq process both the tri- and dilepton signatures are accompanied by a b quark and a light-flavor forward jet. In addition, a significant $E_{\mathrm{T}}^{\text {miss }}$ can be expected because of the undetected neutrinos from the leptonic $\mathrm{W}$ decays. While the leptonic branching fraction of the $\mathrm{W}$ is relatively small, the presence of multiple leptons and identified $b$ jets in the final state reduces the number of background events. The tHq search in this final state has some acceptance for events where $\tau$ leptons, stemming either from the decay of one or more $\mathrm{W}$ bosons, or from Higgs boson decays, give rise to electrons or muons in the decay chain. Events with hadronically decaying $\tau$ leptons are considered separately in section 3.4 .

The trigger used to select the analysis sample requires the presence of two high- $p_{\mathrm{T}}$ electrons or muons. The $p_{\mathrm{T}}$ thresholds are 17 and $8 \mathrm{GeV}$ for the leading and subleading leptons, respectively. The trigger efficiency for signal events with two high- $p_{\mathrm{T}}$ leptons is higher than $98 \%$, and almost $100 \%$ for those with three leptons.

Various SM processes contribute as background in the signal region: diboson (WZ, $\mathrm{WW}, \mathrm{ZZ}, \mathrm{W}^{ \pm} \mathrm{W}^{ \pm} \mathrm{qq}$ ) and triboson (WWW, WWZ, WZZ) production, associated production of $t \bar{t}$ with a boson ( $t \bar{t} W^{ \pm}, t \bar{t} Z, t \bar{t} H, t \bar{t} \gamma$, and $\left.t \bar{t} \gamma^{*}\right)$, t $\bar{t}$ with two $\mathrm{W}$ bosons ( $t \bar{t} W W$ ), 

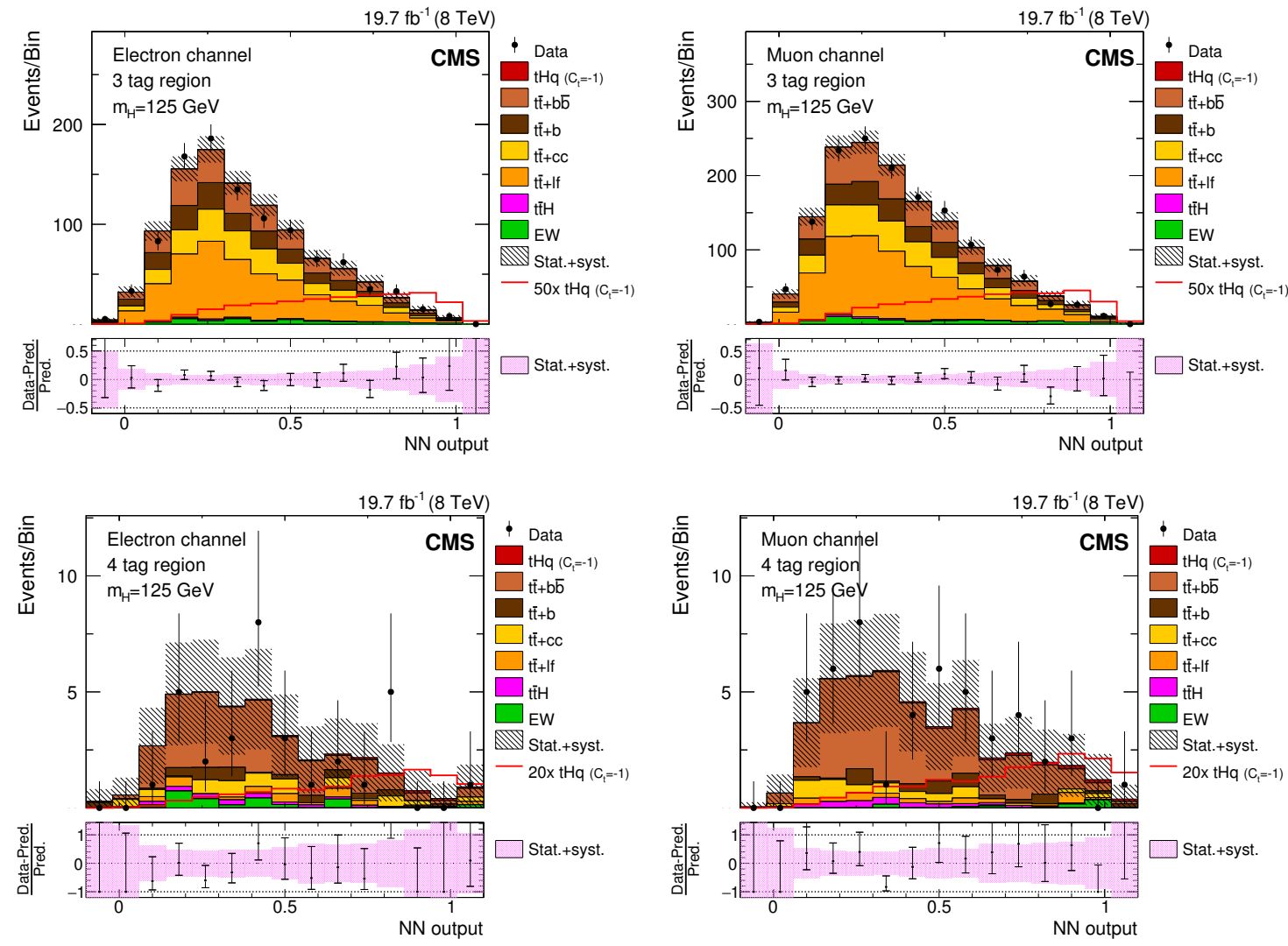

Figure 3. Distributions of the NN output for the $\mathrm{H} \rightarrow \mathrm{b} \overline{\mathrm{b}}$ channel for events with three (four) b-tagged jets are shown in the upper (lower) row. The left (right) column shows events containing a high- $p_{\mathrm{T}}$ electron (muon). All backgrounds are normalized to the output of a maximum likelihood fit of the corresponding distributions. "EW" indicates electroweak backgrounds: single top quark, $\mathrm{W} / \mathrm{Z}$ boson plus jets, and di- and tri-boson production. The line shows the expected contribution from the tHq process with $C_{\mathrm{t}}=-1$ multiplied by the factor indicated in the legend. In the box below each distribution, the ratio of the observed and predicted event yields is shown. The shaded band represents the post-fit systematic and statistical uncertainties.

single top quark associated production with a $\mathrm{Z}$ boson $(\mathrm{tZq})$, and production of same-sign $\mathrm{W}$ bosons via double parton scattering (WW).

\subsubsection{Background modeling}

While diboson backgrounds are produced with a relatively large cross section, their contribution is strongly reduced by imposing a veto on lepton pairs compatible with a $\mathrm{Z}$ boson decay in the trilepton channel ( $\mathrm{Z}$ boson veto) or by vetoing additional leptons in the event in the same-sign dilepton channel. Furthermore, the requirement of a b-tagged or forward jet suppresses contributions from diboson processes. The rate of DY events is strongly reduced by the $\mathrm{Z}$ boson veto (trilepton channel) and the third lepton veto (same-sign dilepton channel). Triboson production has a very small cross section and is further reduced by rejecting events with extra leptons. In addition, both diboson and triboson production do not generally include forward jets or jets from b quark decays. 
Associated production of $t \bar{t}$ and vector bosons ( $\left.t \bar{t} W^{ \pm}, t \overline{t Z}\right)$ or Higgs boson ( $\left.t \bar{t} \mathrm{H}\right)$, although having fairly small cross sections, have high lepton and jet multiplicities as well as two final-state b quarks and can contribute significantly. Because of its very large cross section, $\mathrm{t} \overline{\mathrm{t}}$ production is expected to be the major source of background for both channels, when additional leptons are produced in the decay of B hadrons or when light jets are misidentified as leptons.

An additional background in the case of same-sign dileptons arises when the charge of a lepton in events with an opposite-sign lepton pair is misidentified. This happens not so much because of track misreconstruction, but rather because of strongly asymmetric conversions of hard bremsstrahlung photons emitted from the initial lepton, and is therefore much more likely to occur for electrons than for muons. In the case where the original electron loses most of its energy to the radiated photon, and the conversion daughter with opposite charge carries most of the momentum, the resulting track can have opposite curvature to the original lepton. Furthermore, the same-sign channel has a contribution from the associated production of two same-sign $\mathrm{W}$ bosons and two light quark jets, $\mathrm{W}^{ \pm} \mathrm{W}^{ \pm} \mathrm{qq}$.

Backgrounds involving nonprompt leptons and charge misidentification are estimated using data-driven methods. All the remaining processes are estimated from MC simulation, corrected for data/MC scale factors and pileup distribution, using NLO cross sections where available.

The "tight-to-loose" method is used for estimating the t $\overline{\mathrm{t}}$ background. It is based on defining two lepton selection levels: the tight criteria, corresponding to the full lepton identification used in the signal selection; and a looser selection designed to accept more background leptons. The probability of a nonprompt lepton to pass the tight cut after passing the loose cut (the tight-to-loose rate, $f$ ) is then extracted from data control samples. Nonprompt leptons include real leptons from heavy flavor hadron decays, jets from light quarks misreconstructed as leptons, as well as photon conversions. Finally, the signal selection is extended with the loose lepton selection, and the additional event yield is weighted to arrive at an estimate for the expected contribution from nonprompt leptons. Events with one tight and one loose lepton obtain a weight of $f /(1-f)$, whereas events with two loose leptons are weighted by $-f_{1} f_{2} /\left(\left(1-f_{1}\right)\left(1-f_{2}\right)\right)$. The method assumes $f$ to be consistent between signal and control samples, and that there are only two categories of leptons with consistent efficiencies of passing the tight selection: prompt leptons from $\mathrm{W}$ and $\mathrm{Z}$ boson decays, and nonprompt leptons.

The tight-to-loose rate is defined as the ratio between $N_{\text {tight }}$ and $N_{\text {loose }}$, where $N_{\text {loose }}$ is the number of candidate leptons that pass the loose selection, based on relaxed isolation and impact parameter requirements, and $N_{\text {tight }}$ is the number of loose leptons that also fulfill the tight requirements defined in the analysis. The rate $f$ is measured in a data sample enriched in background leptons, and parametrized as a function of the $p_{\mathrm{T}}, \eta$, and lepton flavor. For the lepton selections used, the electron tight-to-loose rate varies in the range $1-13 \%$, whereas the muon rate varies between $5-23 \%$.

Similarly, the contribution of events with a misidentified lepton charge to the samesign channel is estimated using the charge misidentification probability and the yield of opposite-sign pairs in the signal selection. The electron charge misidentification proba- 
bility is extracted from an independent data sample based on $\mathrm{Z}$ boson decays, and cross checked with expectations from $\mathrm{MC}$ simulation. It is binned in $p_{\mathrm{T}}$ and $\eta$, and ranges from about $0.03 \%$ in the barrel to between $0.08 \%$ and $0.28 \%$ in the endcap. The muon charge misidentification probability in the relevant $p_{\mathrm{T}}$ range is negligible.

\subsubsection{Event selection and signal extraction}

A relatively loose selection is applied to maintain a large signal efficiency while suppressing the main backgrounds. For the dilepton analysis, the presence of two same-sign leptons with $p_{\mathrm{T}}>20 \mathrm{GeV}$ and invariant mass $m_{\ell \ell}>20 \mathrm{GeV}$ is required. No additional leptons can be present in the event. At least one central jet with $p_{\mathrm{T}}>25 \mathrm{GeV}$ is required to be tagged with the CSV algorithm using a loose working point. The event must also contain at least one forward jet $(|\eta|>1.0)$ and an additional central jet $(|\eta|<1.0)$, both with $p_{\mathrm{T}}>25 \mathrm{GeV}$.

For the trilepton analysis, the thresholds for the three lepton transverse momenta are $p_{\mathrm{T}}>20,10$, and $10 \mathrm{GeV}$. To suppress contamination from DY events, the reconstructed dilepton invariant mass closest to the $\mathrm{Z}$ boson mass $\left(m_{\mathrm{Z}}\right)$ must respect the constraint $\left|m_{\ell \ell}-m_{Z}\right|>15 \mathrm{GeV}$. The presence of large missing transverse energy suggests the presence of multiple neutrinos, hence a cut on $E_{\mathrm{T}}^{\text {miss }}>30 \mathrm{GeV}$ is applied. Only events with one jet with $p_{\mathrm{T}}>25 \mathrm{GeV}$ and $|\eta|<2.4$ tagged with the medium working point of the CSV algorithm are selected, and at least one forward jet with $p_{\mathrm{T}}>25 \mathrm{GeV}$ and $|\eta|>1.5$ must be present.

The production cross section times branching fraction for the signal (assuming $C_{\mathrm{t}}=-1$ ) is just a few fb, resulting in a fairly small signal-to-background fraction even for a tight selection. Therefore a multivariate analysis method is used to build a Bayes classifier as in eqs. (3.1) and (3.2) to further reduce backgrounds. A search for an optimal set of variables is performed, to find those that best separate the signal and the backgrounds. The discriminating variables can be put into three broad categories: forward activity, jet and b jet multiplicity, and lepton kinematic properties and charge. The variables to enter the classifier are chosen to be minimally correlated while providing good discrimination power.

For the same-sign lepton final state, the following set of variables has been chosen: the scalar sum of the $p_{\mathrm{T}}$ of all the jets; the jet multiplicity; the medium b-tagged jet multiplicity; the $|\eta|$ value of the leading jet with $|\eta|>1.0$; the $\Delta \eta$ value between the most forward jet and second-most forward jet or lepton; the charge of the leptons; the azimuthal angle difference between the two leptons $\left(\Delta \phi_{\ell \ell}\right)$; and the $p_{\mathrm{T}}$ of the trailing lepton.

In the case of the trilepton final state the selected variables are: the multiplicity of untagged central jets (with $|\eta|<1.5$ ); the number of forward jets with $|\eta|>2.4$; the total sum of the charges of the three leptons; the minimum value of $\Delta R$ between the leptons in the event; and the $\Delta \eta$ value between the b-tagged jet and the most forward jet.

To derive an upper limit on the signal production cross section for $C_{\mathrm{t}}=-1$, a maximum likelihood fit of the classifier output is then performed in all three channels. Table 2 shows the observed data yields and the post-fit expected number of signal and background events, 


\begin{tabular}{|c|c|c|c|}
\hline Process & $\mathrm{e} \mu$ & $\mu \mu$ & $\ell \ell \ell$ \\
\hline $\mathrm{tH}(\tau \tau) \mathrm{W}\left(C_{\mathrm{t}}=-1\right)$ & $0.13 \pm 0.14$ & $0.10 \pm 0.12$ & $0.12 \pm 0.12$ \\
\hline $\mathrm{tH}(\mathrm{WW}) \mathrm{W}\left(C_{\mathrm{t}}=-1\right)$ & $0.47 \pm 0.48$ & $0.28 \pm 0.29$ & $0.35 \pm 0.35$ \\
\hline $\mathrm{tH}(\tau \tau) \mathrm{q}\left(C_{\mathrm{t}}=-1\right)$ & $0.90 \pm 0.91$ & $0.59 \pm 0.61$ & $0.56 \pm 0.58$ \\
\hline $\mathrm{tH}(\mathrm{WW}) \mathrm{q}\left(C_{\mathrm{t}}=-1\right)$ & $3.73 \pm 3.84$ & $2.55 \pm 2.62$ & $1.73 \pm 1.80$ \\
\hline Total signal $\left(C_{\mathrm{t}}=-1\right)$ & $5.22 \pm 3.98$ & $3.53 \pm 2.71$ & $2.76 \pm 1.93$ \\
\hline $\mathrm{W}^{ \pm} \mathrm{W}^{ \pm} \mathrm{qq}$ & $6.03 \pm 0.85$ & $4.60 \pm 0.68$ & - \\
\hline $\mathrm{WZ}, \mathrm{WW}, \mathrm{ZZ}$ & $8.83 \pm 3.25$ & $5.47 \pm 2.10$ & $1.19 \pm 0.14$ \\
\hline Rare SM bkg. & $2.57 \pm 1.23$ & $1.40 \pm 0.68$ & $0.11 \pm 0.03$ \\
\hline $\mathrm{t} \overline{\mathrm{t}} \gamma^{*}$ & $1.04 \pm 0.42$ & $0.50 \pm 0.20$ & - \\
\hline $\mathrm{t} \overline{\mathrm{t}} \gamma$ & $2.02 \pm 0.60$ & $0.09 \pm 0.03$ & - \\
\hline $\mathrm{t} \overline{t Z}$ & $2.87 \pm 0.50$ & $2.23 \pm 0.41$ & $2.21 \pm 0.36$ \\
\hline $\mathrm{t} \bar{t} \mathrm{~W}^{ \pm}$ & $14.85 \pm 3.32$ & $10.18 \pm 2.24$ & $3.03 \pm 0.51$ \\
\hline $\mathrm{t} \overline{\mathrm{t}} \mathrm{H}$ & $3.24 \pm 0.47$ & $2.26 \pm 0.34$ & $1.52 \pm 0.18$ \\
\hline Charge misid & $6.96 \pm 1.76$ & - & - \\
\hline Nonprompt & $63.7 \pm 12.5$ & $33.3 \pm 8.3$ & $31.4 \pm 6.5$ \\
\hline Total background & $112.1 \pm 13.5$ & $60.1 \pm 9.0$ & $39.5 \pm 6.6$ \\
\hline Data & 117 & 66 & 42 \\
\hline
\end{tabular}

Table 2. Data yields and post-fit expected backgrounds after the event pre-selection for single top plus Higgs events appearing in events with $\mathrm{e} \mu, \mu \mu$, or $\ell \ell \ell$. Contributions from tHq and tHW are shown separately, as well as expected events where the Higgs boson decays to W bosons, or to tau leptons. Uncertainties include systematic and statistical sources. "Rare SM" comprises VVV, tbZ,

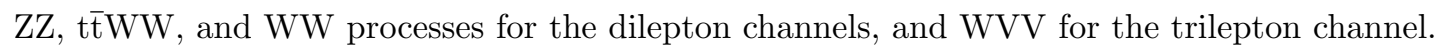

where the trilepton channel, $\ell \ell \ell$, consists of eee, ee $\mu, \mu \mu \mathrm{e}$, and $\mu \mu \mu$ final states. The post-fit classifier output for all the channels is shown in figure 4 .

\section{$3.4 \mathrm{H} \rightarrow \tau^{+} \tau^{-}$channel}

The previous section presented a strategy for identifying tHq events that captured events with leptonically decaying tau leptons. An orthogonal strategy is devised to analyze signal events with a reconstructed tau lepton, through its decay to hadrons $\left(\tau_{\mathrm{h}}\right)$. The analysis described here is based on two final states with three reconstructed leptons, $\mathrm{e} \mu \tau_{\mathrm{h}}$ and $\mu \mu \tau_{\mathrm{h}}$. These final states are chosen to select signal events where the two $\tau$ leptons from the Higgs boson decay give rise to an $\mathrm{e} \tau_{\mathrm{h}}$ or $\mu \tau_{\mathrm{h}}$ final state and the top quark decay produces the third lepton.

The major SM background processes that can lead to the same lepton final state include $\mathrm{WZ}, \mathrm{ZZ}, \mathrm{t} \overline{\mathrm{t}} \mathrm{H}$, and $\mathrm{t} \overline{\mathrm{t}}+\mathrm{W} / \mathrm{Z}$ production. The contributions from reducible backgrounds include $t \bar{t}$, single top, $\mathrm{W}+$ jets, $\mathrm{Z}+$ jets, and multijet production. These contributions are estimated using events from control samples in the data. 

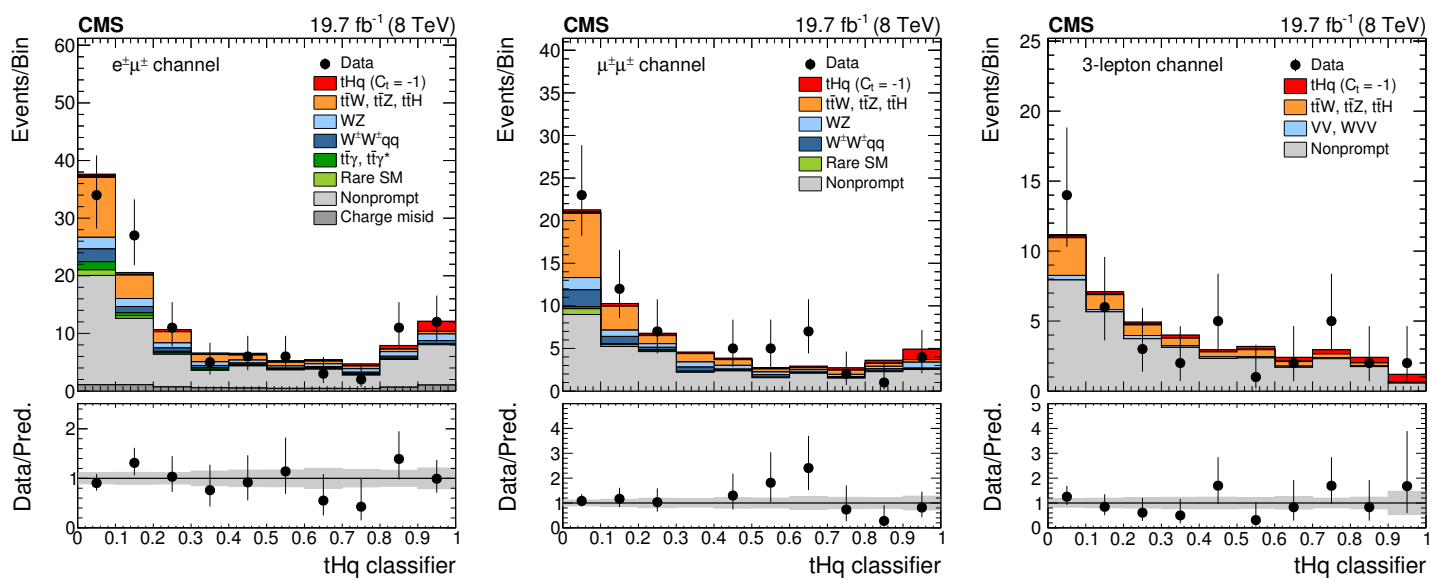

Figure 4. Post-fit Bayes classifier output, for the e $\mu$ (left), $\mu \mu$ (center), and trilepton channel (right). In the box below each distribution, the ratio of the observed and predicted event yields is shown. The gray band represents the post-fit systematic and statistical uncertainties.

\subsubsection{Event selection}

Candidate events are collected using either e $\mu$ or $\mu \mu$ triggers, depending on the final state. In the final event selection, the leading (subleading) electron or muon is required to have $p_{\mathrm{T}}^{\ell}>20(10) \mathrm{GeV}$. Electrons (muons) are required to have $|\eta|<2.5(2.4)$ and to pass basic identification requirements. To suppress secondary leptons from b-flavored hadron decays, an isolation classifier is computed with boosted decision trees using variables based on impact parameters with respect to the reconstructed primary interaction vertex (defined as the vertex with highest $\sum p_{\mathrm{T}}^{2}$ of its associated tracks), variables related to the isolation of the lepton, and variables related to the reconstructed jet closest to each lepton [10].

In addition, either the electron and muon in the $\mathrm{e} \mu \tau_{\mathrm{h}}$ final state or the two muons in the $\mu \mu \tau_{\mathrm{h}}$ final state are required to have equal charges. This same-sign requirement suppresses contributions from backgrounds with prompt opposite-sign dileptons and additional jets that can be misidentified as hadronic tau leptons, such as $\mathrm{Z} / \gamma^{*} \rightarrow \mu^{+} \mu^{-}+$fake $\tau$ jet and $\mathrm{t} \overline{\mathrm{t}} / \mathrm{Z} \rightarrow \tau \tau+$ fake $\tau$ jet.

The selected leptons $\left(\mathrm{e}, \mu\right.$, and $\left.\tau_{\mathrm{h}}\right)$ are required to be at least 0.5 apart in $\Delta R$. To reduce contributions from $\mathrm{ZZ}$ and $\mathrm{t} \overline{\mathrm{t}} \mathrm{Z}$ backgrounds, events with additional isolated electrons and muons are rejected.

The $\tau_{\mathrm{h}}$ candidate, reconstructed as described in section 2 , is required to have $p_{\mathrm{T}}>$ $20 \mathrm{GeV},|\eta|<2.3$, and to pass identification and isolation criteria to reject misidentified $\tau_{\mathrm{h}}$ candidates from jets, electrons, or muons [8]. The charge of the $\tau_{\mathrm{h}}$ candidate is required to be opposite to that of other leptons, e or $\mu$. To suppress background events without $\mathrm{b}$ quark jets, the presence of at least one jet with $p_{\mathrm{T}}>20 \mathrm{GeV}$ and $|\eta|<2.4$ identified as coming from a b quark with the medium working point of the CSV algorithm is required. This requirement particularly reduces the contamination from $\mathrm{Z} \rightarrow \tau \tau+$ jets backgrounds.

\subsubsection{Background modeling}

The signal processes as well as irreducible background processes with the same lepton final state are modeled using simulated events. These irreducible backgrounds include WZ, ZZ, 
$\mathrm{t} \overline{\mathrm{t}} \mathrm{H}$, and $\mathrm{t} \overline{\mathrm{t}}+\mathrm{W} / \mathrm{Z}$ production. The simulation is corrected for differences between data and simulation, including the distribution of pileup interactions, the efficiencies for the leptons to pass trigger, identification, and isolation criteria, and the identification efficiency for b quark jets.

The contributions from reducible background processes are estimated using a similar tight-to-loose method as discussed in section 3.3.1. The contributions from events where either the charge of an electron or muon is misreconstructed or the $\tau_{\mathrm{h}}$ candidate is misidentified are negligible. Therefore, three control samples are defined where one or both leptons fail the tight identification and isolation criteria, but pass all other selections: (i) one of the leptons fails the tight criteria; (ii) the other lepton fails the tight criteria; and (iii) both leptons fail the tight criteria.

The tight-to-loose rates $(f)$ for jets misidentified as electrons or muons are measured in control regions enriched in $\mathrm{W}+$ jets and $\mathrm{t} \overline{\mathrm{t}}$ events. The selection criteria for these control regions differ from the signal selection by requiring the transverse mass of the leading isolated $\ell-E_{\mathrm{T}}^{\text {miss }}$ system to be greater than $35 \mathrm{GeV}$ and by requiring that there be no selected $\tau_{\mathrm{h}}$ leptons, making the selection orthogonal to the signal sample. The rate $f$ is parameterized based on the lepton $p_{\mathrm{T}}$ and the number of jets with $p_{\mathrm{T}}>20 \mathrm{GeV}$ in the event, using the $k$-nearest neighbor algorithm [48]. The small contributions from genuine isolated leptons from $\mathrm{WZ}, \mathrm{ZZ}$, and $\mathrm{t} \overline{\mathrm{t}}+\mathrm{Z} / \mathrm{W}$ events in the control region are estimated using simulated samples and subtracted.

For two leptons $\ell_{1}$ and $\ell_{2}$ with rates $f_{1}$ and $f_{2}$, the spectra of the reducible background contributions in the signal region are estimated by weighting events in the regions where only $\ell_{1}$ or $\ell_{2}$ fail the tight selection criteria by $f_{1}$ or $f_{2}$, respectively, and events in the region where both leptons fail the tight selection criteria by $-f_{1} f_{2}$. This procedure is conceptually identical to the one described in section 3.3.1. The reducible background estimation is validated in a control region where the $\tau_{\mathrm{h}}$ candidates fail the tight isolation criteria.

\subsubsection{Signal extraction}

To extract the signal contribution, a multivariate method is used that combines the discrimination power of several variables. The signal extraction is performed with a linear discriminant, also known as Fisher discriminant, as implemented in the TMVA package [48]. Because of the small number of simulated and estimated background events in the signal region, the Fisher discriminant is trained using events from a control region with the $\tau$ isolation criteria inverted. This provides a sufficient number of training events and avoids overtraining from the events in the signal region, thereby improving the final expected sensitivity of the analysis.

The Fisher discriminant is trained using ten input variables making use of (i) the forward jet present in tHq production, (ii) the expectation of only one b quark jet as

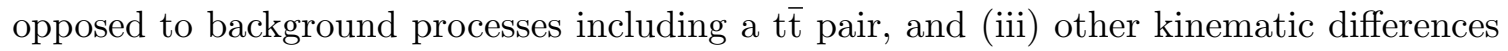
between the tHq and the background processes.

The training variables are: $|\eta|$ of the jet with the largest $|\eta|$ value and $p_{\mathrm{T}}>20 \mathrm{GeV}$, $|\eta|$ of the jet with the largest $|\eta|$ value and $p_{\mathrm{T}}>30 \mathrm{GeV}$, "centrality" (the ratio of the $p_{\mathrm{T}}$ sum of all selected objects and the energy sum), number of $\mathrm{b}$ jets, $p_{\mathrm{T}}$ of the leading $\mathrm{b}$ jet, 

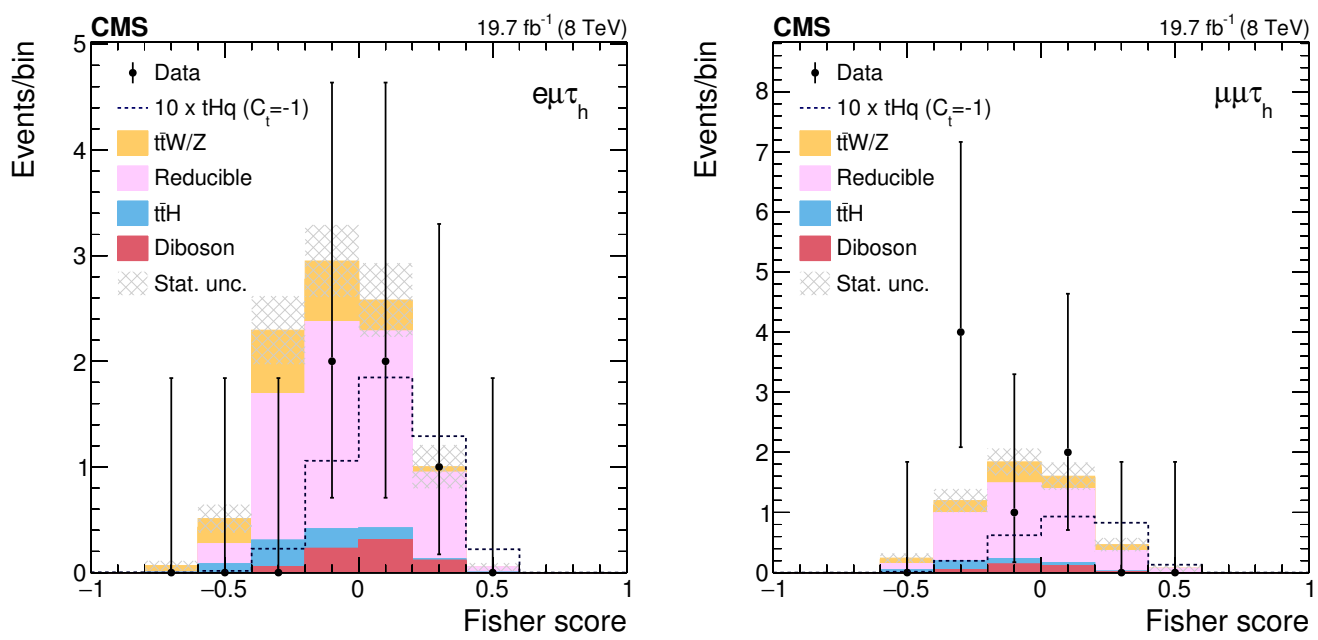

Figure 5. Expected (histograms) and observed (points) distributions of the Fisher discriminant in the $\mathrm{e} \mu \tau_{\mathrm{h}}$ channel (left) and $\mu \mu \tau_{\mathrm{h}}$ channel (right). The dashed line gives the expected contribution from the tHq signal $\left(C_{\mathrm{t}}=-1\right)$ case, multiplied by ten.

\begin{tabular}{|ccc|}
\hline Process & $\mathrm{e} \mu \tau_{\mathrm{h}}$ & $\mu \mu \tau_{\mathrm{h}}$ \\
\hline $\mathrm{tHq}\left(C_{\mathrm{t}}=-1\right)$ & $0.42 \pm 0.05$ & $0.26 \pm 0.03$ \\
$\mathrm{tHW}\left(C_{\mathrm{t}}=-1\right)$ & $0.06 \pm 0.01$ & $0.04 \pm 0.01$ \\
\hline $\mathrm{t} \overline{\mathrm{t} H}$ & $0.6 \pm 0.1$ & $0.3 \pm 0.1$ \\
$\mathrm{t} \overline{\mathrm{tV}}$ & $1.8 \pm 0.4$ & $0.9 \pm 0.2$ \\
$\mathrm{VV}$ & $0.7 \pm 0.1$ & $0.3 \pm 0.1$ \\
Reducible & $6.3 \pm 3.1$ & $4.5 \pm 1.9$ \\
\hline Total background & $9.5 \pm 3.7$ & $5.4 \pm 2.4$ \\
\hline Data & 5 & 7 \\
\hline
\end{tabular}

Table 3. Expected and observed event yields for the $\mathrm{e} \mu \tau_{\mathrm{h}}$ and $\mu \mu \tau_{\mathrm{h}}$ channels. The given uncertainties include all systematic uncertainties added in quadrature, including uncertainties due to the limited numbers of simulated events or events in control data samples.

number of jets with $p_{\mathrm{T}}>30 \mathrm{GeV}$, e $\tau_{\mathrm{h}}$ invariant mass ( $\mu \tau_{\mathrm{h}}$ mass with the leading muon in the $\mu \mu \tau_{\mathrm{h}}$ channel), $\mu \tau_{\mathrm{h}}$ mass ( $\mu \tau_{\mathrm{h}}$ mass with the subleading muon in the $\mu \mu \tau_{\mathrm{h}}$ channel), $\mathrm{e} \mu$ mass ( $\mu \mu$ mass in the $\mu \mu \tau_{\mathrm{h}}$ channel), and $E_{\mathrm{T}}^{\text {miss }}$. The training is performed assuming the tHq process as a signal and the rest of the processes as background. The tHW process is not considered as a part of the signal in the training because of its background-like shape, but considered as a part of signal for the signal extraction.

The signal extraction is performed using a combined maximum likelihood fit of the Fisher discriminant distributions in the two channels. Figure 5 shows the final distributions of the discriminant in the e $\mu \tau_{\mathrm{h}}$ and $\mu \mu \tau_{\mathrm{h}}$ categories. The expected and observed yields in all categories are given in table 3 . 


\section{Systematic uncertainties}

Various sources of systematic uncertainty influence the upper limit on the tHq production cross section. In general, the systematic uncertainties can introduce rate uncertainties on a specific process as well as shape uncertainties on the distribution from which the upper limit on the process is finally derived. These uncertainties are handled by means of nuisance parameters, which are allowed to float during the limit setting procedure.

The uncertainty in the trigger efficiencies translates into an uncertainty in the final rates of up to $5 \%$.

The uncertainty from the jet energy scale [36] is evaluated by varying the energy scale for all jets in the signal and background simulation simultaneously within their uncertainty as a function of jet $p_{\mathrm{T}}$ and $\eta$, and re-evaluating the yields and discriminant shapes of all processes. The limitations on the knowledge of the jet energy scale lead to an uncertainty that in some channels can be as large as $8 \%$. Jet energy resolution uncertainties have a smaller effect, up to $3 \%$ in the event yields.

The corrections for the $\mathrm{b}$ tagging efficiencies for light-flavored, $\mathrm{c}$, and $\mathrm{b}$ quark jets have associated uncertainties [38], which are parameterized as a function of the $p_{\mathrm{T}}, \eta$, and flavor of the jets. Their effect on the analysis is evaluated by shifting the correction factor of each jet up and down within their measured uncertainty.

For photon identification, the uncertainty in the data/MC efficiency scale factor from the fiducial region determines the overall uncertainty, as measured using a tag-and-probe technique applied to $\mathrm{Z} \rightarrow$ ee events (3.0\% in the ECAL barrel, $4.0 \%$ in ECAL endcap) [49]. For the uncertainties related to the photon energy scale and resolution, the photon energy is shifted and smeared, respectively, within the known uncertainty for photons [50].

The cross sections used to estimate signal and background rates, where applicable, are of at least NLO accuracy and have associated uncertainties arising primarily from the PDFs and the choice of the factorization and renormalization scales.

The effect from the PDF uncertainties has been evaluated on signal and backgrounds following the PDF4LHC prescription [51, 52], and ranges from 1 to $8 \%$ depending on the quark or gluon nature of the colliding partons. The effect of changing renormalization and factorization scales is evaluated for both signal and backgrounds by changing them simultaneously up and down by factors of two, producing effects on rates extending up to $13 \%$ for $\mathrm{t} \overline{\mathrm{t}} \mathrm{H}$ production. For the $\mathrm{H} \rightarrow \gamma \gamma$ and $\mathrm{H} \rightarrow \mathrm{WW}$ analyses, where the signal is modeled using the five-flavor scheme, the overall event selection efficiency is re-evaluated using a sample simulated with the four-flavor scheme. The corresponding change in signal selection efficiency is taken as a systematic uncertainty, and is $5.5 \%$ in the diphoton and up to $16 \%$ in the multilepton channels.

The large $t \bar{t}$ background in the $\mathrm{H} \rightarrow \mathrm{b} \overline{\mathrm{b}}$ final state requires a special treatment of the $t \bar{t}+$ jets background component, which is split into four different categories, depending on the flavor of the additional final state partons: $t \bar{t}+b \bar{b}, t \bar{t}+b, t \bar{t}+1 / 2 c$, and $t \bar{t}+$ light flavors. Each of the $t \bar{t}+$ heavy flavor components receives a conservative $50 \%$ rate uncertainty in addition to what is assigned to the t $\overline{\mathrm{t}}$ background rate uncertainties. Dedicated MADGRAPH+PYTHIA samples with varied renormalization and factorization scales and with varied matching thresholds are used to introduce additional nuisance parameters, which 
can alter the rate and the shape of the t⿱亠t backgrounds. Reweighting the top quark $p_{\mathrm{T}}$ distribution for $t \bar{t}$ events needs to be accounted for by a separate rate and shape systematic uncertainty [53]. The systematic uncertainty arising from scale variations in the sample generation is also taken into account for the signal process. For the statistical uncertainties, bin-by-bin uncertainties in the NN output shape are taken into account.

Uncertainties in the efficiencies for lepton identification, isolation and impact parameter requirements are estimated by comparing variations in the difference in performance between data and $\mathrm{MC}$ simulation using a high-purity sample of $\mathrm{Z}$ boson decays with a tag-and-probe method. These uncertainties vary between 1 and $5 \%$, depending on the lepton flavor and selection. The overall uncertainty is about $5 \%$ per lepton for the same-sign dilepton final state, while it is $1.6 \%$ in the case of the trilepton final state. For trigger efficiencies, no scale factors are used on simulation to correct for possible differences between data and MC, assuming a trigger efficiency of $100 \%$ for the double-lepton triggers. The uncertainty in the yields derived from simulation due to the trigger efficiency is about $1 \%$.

The uncertainty in the misidentification probabilities for nonprompt leptons is estimated from simulation for the same-sign dilepton final state. The misidentification rate is estimated following the same approach and parameterization used in the multijetdominated control sample, but using instead MC samples with a similar composition. This simulation-based misidentification rate is then applied to MC samples with the expected background composition in the signal sample, and the amount of disagreement between the number of nonprompt leptons predicted by the parameterized misidentification rate and those actually observed in this collection of MC samples is used to estimate the systematic uncertainty. In the case of the same-sign dilepton final state, the uncertainty is assessed separately for different $p_{\mathrm{T}}, \eta$, and b-tagged jet multiplicity bins for each flavor. The overall uncertainty amounts to about $40 \%$, which is applied using linear and quadratic deformations of the $p_{\mathrm{T}^{-}}$and $\eta$-dependent misidentification rate. For the trilepton final state, a similar method is used to estimate a total rate uncertainty of $30 \%$. Additional sources of uncertainty for this final state are considered. The first contribution comes from the change in the tight-to-loose rate as a result of applying a requirement on the $E_{\mathrm{T}}^{\mathrm{miss}}$ in the multijet control region used to estimate this rate, changing the diboson background contribution. The overall effect on the final prediction is about $10 \%$. The second contribution is studied by changing the measured tight-to-loose rate up and down within its statistical uncertainty and propagated to the final weight estimation. The total effect on the expected number of events is about $14 \%$.

In the $\mathrm{H} \rightarrow \tau \tau$ analysis, an uncertainty of $50 \%$ is assigned to the yield of reducible backgrounds, uncorrelated between channels and categories. This arises from the sum of the uncertainties in the estimation of the nonprompt rates with and without the requirement of a b-tagged jet, the agreement of the predicted and observed event yields in control regions, and the comparison with simulated $t \bar{t}$ events. An uncertainty of $6 \%$ is assigned to the $\tau_{\mathrm{h}}$ identification efficiency [37]. The $\tau_{\mathrm{h}}$ energy scale uncertainty is $3 \%$ [8]; this propagates to an uncertainty of comparable size in the simulated yields.

The uncertainty in the integrated luminosity, which is common to all of the channels, is $2.6 \%$ [54]. 


\begin{tabular}{|ccccc|}
\hline tHq channel & Best-fit $\mu$ & \multicolumn{4}{c|}{$95 \%$ CL upper limits on $\mu=\sigma / \sigma_{C_{\mathrm{t}}=-1}$} \\
\hline & Observed & \multicolumn{4}{c|}{ Expected } \\
& & Median & $68 \%$ CL range & $95 \%$ CL range \\
$\gamma \gamma$ & 4.1 & 4.1 & {$[3.7,4.2]$} & {$[3.4,5.3]$} \\
$\mathrm{b} \overline{\mathrm{b}}$ & 7.6 & 5.4 & {$[3.8,7.7]$} & {$[2.8,10.7]$} \\
Multilepton & 6.7 & 5.0 & {$[3.6,7.1]$} & {$[2.9,10.3]$} \\
$\tau \tau$ & 9.8 & 11.4 & {$[8.1,16.7]$} & {$[6.0,24.9]$} \\
\hline Combined & 2.8 & 2.0 & {$[1.6,2.8]$} & {$[1.2,4.1]$} \\
\hline
\end{tabular}

Table 4. Upper limit on $\mu=\sigma / \sigma_{C_{\mathrm{t}}=-1}$ for each tHq channel. The observed and expected $95 \% \mathrm{CL}$ upper limits on the signal strength parameter $\mu$ for each tHq channel are also shown.

\section{Results}

No significant excess of events over the expectations is observed in the different channels, and the observed yields and predicted backgrounds are used to set limits on the tHq production cross section. A binned likelihood spanning all analysis channels included in a given result is constructed. Uncertainties in the signal and background predictions are incorporated by means of nuisance parameters.

Limits are computed using the modified-frequentist $\mathrm{CL}_{\mathrm{s}}$ method $[55,56]$. Results are obtained independently for each of the distinct tHq signatures (diphoton, $b \bar{b}, \mathrm{WW}$, and $\tau \tau)$ as well as combined. There is no significant deviation in the data from the predicted event yields.

The $C_{\mathrm{t}}=-1$ scenario predicts an enhancement in the tHq production cross section, and in the diphoton branching fraction of the Higgs boson. As a result, the presence of such a signal in the data would be highlighted in the diphoton channel by an enhancement of the yields in all Higgs boson production modes.

The median expected $95 \%$ confidence level (CL) upper limit computed from the combination of all channels is 2.0 times the event yields predicted by negative Yukawa couplings. The corresponding observed upper limit is 2.8. Table 4 shows the limits obtained in the several subchannels, and from the overall combination. Figure 6 provides a visual display of the results.

The 95\% CL expected and observed upper limits on the tHq production cross section are shown in figure 7 as a function of the assumed branching fraction for a Higgs boson decaying to two photons. The latter quantity is normalized to the SM predictions. In addition to the median expected limit under the background-only hypothesis, the bands that contain the one and two standard deviation ranges around the median are also shown.

The overall effect of systematic uncertainties on the analysis is to increase the upper limit for $95 \%$ CL exclusion by $\sim 40 \%$, averaged over the range of $\mathrm{BR}(\mathrm{H} \rightarrow \gamma \gamma)$ values investigated.

Expected limits on the tHq production cross section are set, ranging from 450 to $700 \mathrm{fb}$ depending on the assumed diphoton branching fraction of the Higgs boson. The observed limits are slightly less sensitive, ranging from 600 to $1000 \mathrm{fb}$. 


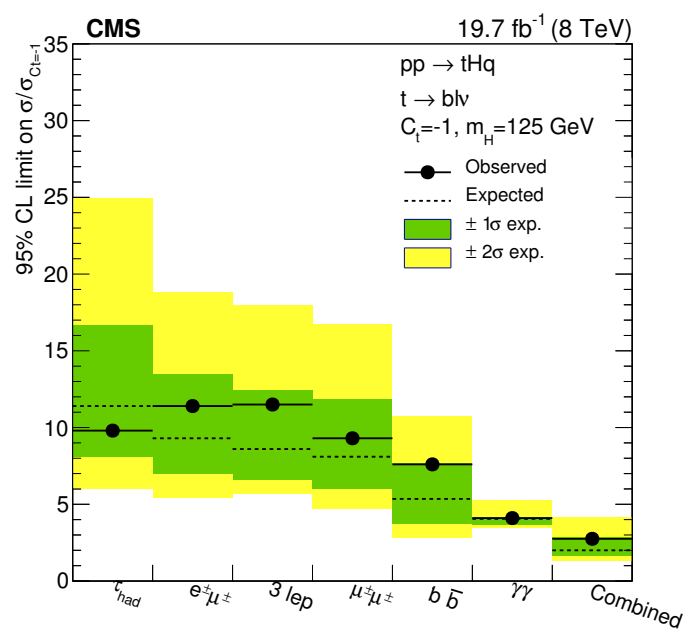

Figure 6. The 95\% CL upper limits on the excess event yields predicted by the enhanced tHq cross section and Higgs boson to diphoton branching fraction for $C_{\mathrm{t}}=-1$. The limits are normalized to the $C_{\mathrm{t}}=-1$ predictions [57], and are shown for each analysis channel, and combined. The black solid and dotted lines show the observed and background-only expected limits, respectively. The $1 \sigma$ and $2 \sigma$ bands represent the 1 and 2 standard deviation uncertainties on the expected limits.

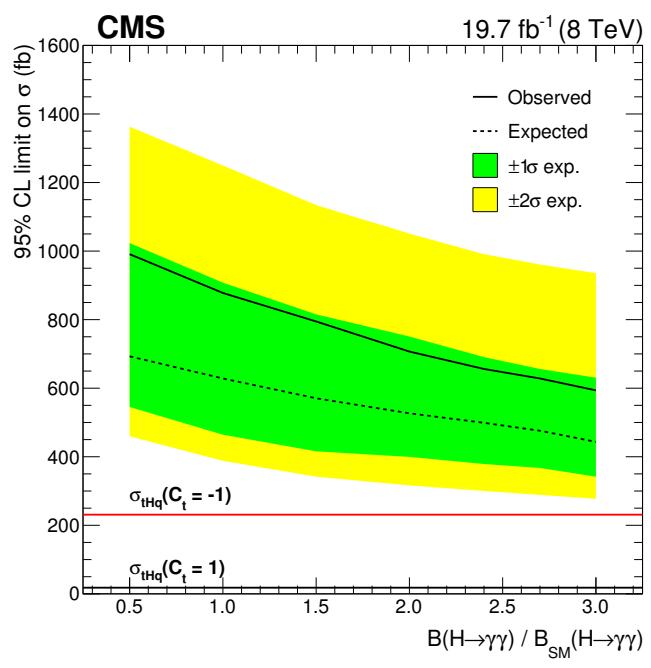

Figure 7. The 95\% CL upper limits on the tHq production cross section as a function of the assumed Higgs boson to diphoton branching fraction. The black solid and dotted lines show the observed and background-only expected limits, respectively. The $1 \sigma$ and $2 \sigma$ bands represent the 1 and 2 standard deviation uncertainties on the expected limits. The red horizontal line shows the predicted tHq cross section for the SM Higgs boson with $m_{\mathrm{H}}=125 \mathrm{GeV}$ in the $C_{\mathrm{t}}=-1$ scenario, while the black horizontal line shows the predicted tHq cross section in the SM (i.e., $C_{\mathrm{t}}=+1$ ).

\section{Summary}

The production of the standard-model-like Higgs boson in association with a single top quark has been investigated using data recorded by the CMS experiment at $\sqrt{s}=8 \mathrm{TeV}$, corresponding to an integrated luminosity of $19.7 \mathrm{fb}^{-1}$. Signatures resulting from leptonic 
top quark decay and different decay modes of the Higgs boson have been analyzed. In particular, the searches have been optimized for the $\mathrm{H} \rightarrow \gamma \gamma, \mathrm{H} \rightarrow \mathrm{b} \overline{\mathrm{b}}, \mathrm{H} \rightarrow \mathrm{WW}$, and $\mathrm{H} \rightarrow \tau \tau$ decay modes. The results are consistent with the background-only hypothesis. $\mathrm{A}$ 95\% confidence level limit on the production cross section of a single top quark plus a Higgs boson with a non-standard-model coupling is set ranging from 600 to $1000 \mathrm{fb}$ depending on the assumed diphoton branching fraction of the Higgs boson. This is the first time that results on anomalous tHq production have been reported. These results can be combined with other Higgs boson measurements to constrain the coupling of the Higgs boson to SM quarks; they can also be used to probe new physics modifying the top-Higgs couplings. The $13 \mathrm{TeV}$ LHC run will allow a precise determination of both the magnitude and the sign of the top quark Yukawa coupling.

\section{Acknowledgments}

We congratulate our colleagues in the CERN accelerator departments for the excellent performance of the LHC and thank the technical and administrative staffs at CERN and at other CMS institutes for their contributions to the success of the CMS effort. In addition, we gratefully acknowledge the computing centers and personnel of the Worldwide LHC Computing Grid for delivering so effectively the computing infrastructure essential to our analyses. Finally, we acknowledge the enduring support for the construction and operation of the LHC and the CMS detector provided by the following funding agencies: BMWFW and FWF (Austria); FNRS and FWO (Belgium); CNPq, CAPES, FAPERJ, and FAPESP (Brazil); MES (Bulgaria); CERN; CAS, MoST, and NSFC (China); COLCIENCIAS (Colombia); MSES and CSF (Croatia); RPF (Cyprus); MoER, ERC IUT and ERDF (Estonia); Academy of Finland, MEC, and HIP (Finland); CEA and CNRS/IN2P3 (France); BMBF, DFG, and HGF (Germany); GSRT (Greece); OTKA and NIH (Hungary); DAE and DST (India); IPM (Iran); SFI (Ireland); INFN (Italy); MSIP and NRF (Republic of Korea); LAS (Lithuania); MOE and UM (Malaysia); CINVESTAV, CONACYT, SEP, and UASLP-FAI (Mexico); MBIE (New Zealand); PAEC (Pakistan); MSHE and NSC (Poland); FCT (Portugal); JINR (Dubna); MON, RosAtom, RAS and RFBR (Russia); MESTD (Serbia); SEIDI and CPAN (Spain); Swiss Funding Agencies (Switzerland); MST (Taipei); ThEPCenter, IPST, STAR and NSTDA (Thailand); TUBITAK and TAEK (Turkey); NASU and SFFR (Ukraine); STFC (United Kingdom); DOE and NSF (U.S.A.).

Individuals have received support from the Marie-Curie program and the European Research Council and EPLANET (European Union); the Leventis Foundation; the A. P. Sloan Foundation; the Alexander von Humboldt Foundation; the Belgian Federal Science Policy Office; the Fonds pour la Formation à la Recherche dans l'Industrie et dans l'Agriculture (FRIA-Belgium); the Agentschap voor Innovatie door Wetenschap en Technologie (IWTBelgium); the Ministry of Education, Youth and Sports (MEYS) of the Czech Republic; the Council of Science and Industrial Research, India; the HOMING PLUS program of the Foundation for Polish Science, cofinanced from European Union, Regional Development Fund; the OPUS program of the National Science Center (Poland); the Compagnia di San Paolo (Torino); the Consorzio per la Fisica (Trieste); MIUR project 20108T4XTM (Italy); 

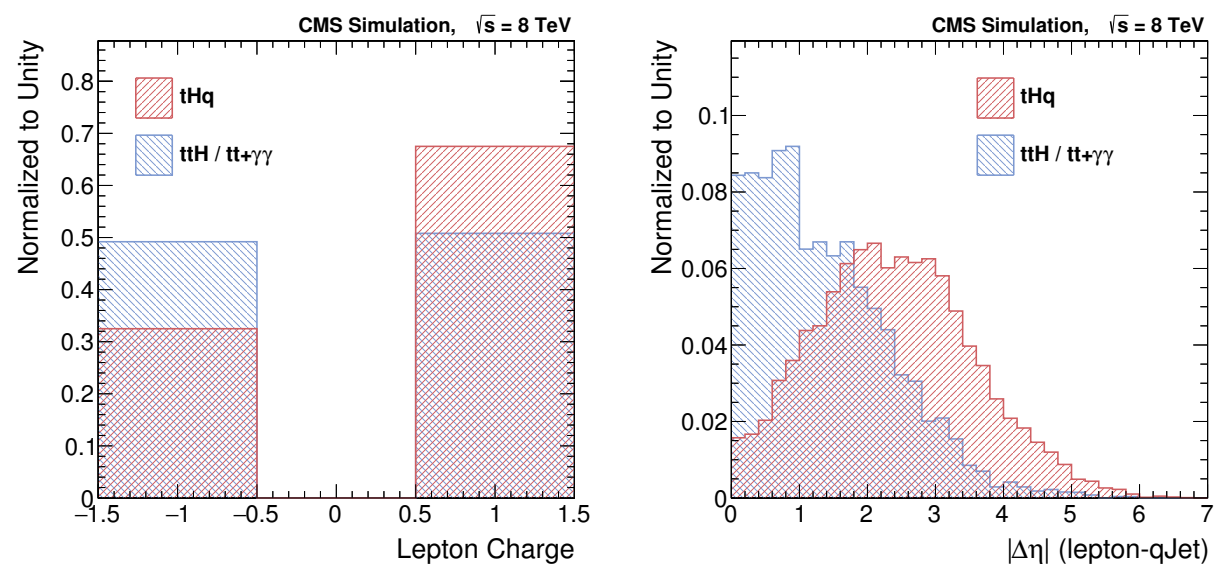

Figure 8. Sample of input variables to the final discriminant in the $\mathrm{H} \rightarrow \gamma \gamma$ analysis. Left: distribution of the electric charge of the reconstructed lepton. Right: distribution of the pseudorapidity difference between the lepton and the forward jet. Both plots are shown after the initial kinematic selection, and both distributions are normalized to unit area.

the Thalis and Aristeia programs cofinanced by EU-ESF and the Greek NSRF; the National Priorities Research Program by Qatar National Research Fund; the Rachadapisek Sompot Fund for Postdoctoral Fellowship, Chulalongkorn University (Thailand); and the Welch Foundation, contract C-1845.

\section{A Selected distributions of inputs to the multivariate discriminants}

The cross section for single top plus Higgs production in proton-proton collisions at $\sqrt{s}=$ $8 \mathrm{TeV}$ is very small, even under the hypothesis of an anomalous Yukawa coupling that is explored in this paper. In addition, the kinematics of the tHq process are only mildly different from the dominant background processes. Multivariate techniques are thus used to improve signal-to-background discrimination. Such techniques are used either at event selection level (in the case of the $\mathrm{H} \rightarrow \gamma \gamma$ analysis) or at signal discrimination level (in the case of $\mathrm{H} \rightarrow \mathrm{WW}$ and $\mathrm{H} \rightarrow \tau \tau$ ) or in multiple steps of the analysis (in the case of $\mathrm{H} \rightarrow \mathrm{b} \overline{\mathrm{b}}$ ). A representative selection of the kinematic distributions that are inputs to the multivariate discriminants are given in this appendix.

One feature common to all analyses is the presence of a charge asymmetry induced by the net positive charge of the proton-proton collisions. This is displayed in the context of the $\mathrm{H} \rightarrow \gamma \gamma$ analysis in figure 8, together with the difference in pseudorapidity between the light-flavor jet and the charged lepton.

In the $\mathrm{H} \rightarrow \mathrm{b} \overline{\mathrm{b}}$ analysis, full event reconstructions are performed under both the tHq and the t $\bar{t}$ hypotheses. Figure 9 shows the invariant mass of the tri-jet system most compatible with the hypothesis of a hadronically-decaying top quark and the transverse momentum of the b-jet pair compatible with originating from the decay of a Higgs boson. Good agreement between data and predictions is observed.

In the $\mathrm{H} \rightarrow \mathrm{WW}$ analysis, the event reconstruction is complicated by the presence of multiple neutrinos. The multiplicity of central jets $(|\eta|<1)$ and forward jets $(|\eta|>1.5)$ 

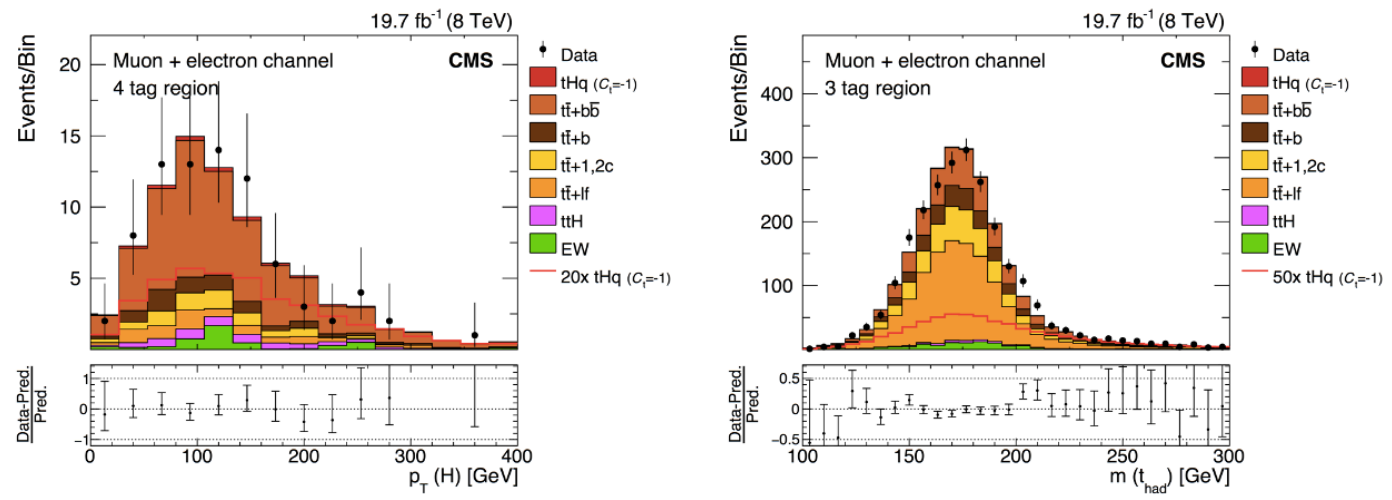

Figure 9. Sample of input variables to the final discriminant in the $\mathrm{H} \rightarrow \mathrm{b} \overline{\mathrm{b}}$ channel. Left: distribution of the reconstructed transverse momentum of the $b \bar{b}$ system in the signal hypothesis for events with an high transverse momentum electron or muon and four b-tagged jets. Right: distribution of the invariant mass of the tri-jet system under the hypothesis of the presence of a hadronically-decaying top quark for electron/muon events with exactly three b-tagged jets. In both cases the signal is normalized to the rate for the analous coupling hypothesis, and is shown stacked on top of the SM background prediction. It is also shown as a separate histogram, magnified by a factor of 20 (50) in the four-tag (three-tag) sample to enhance visibility.
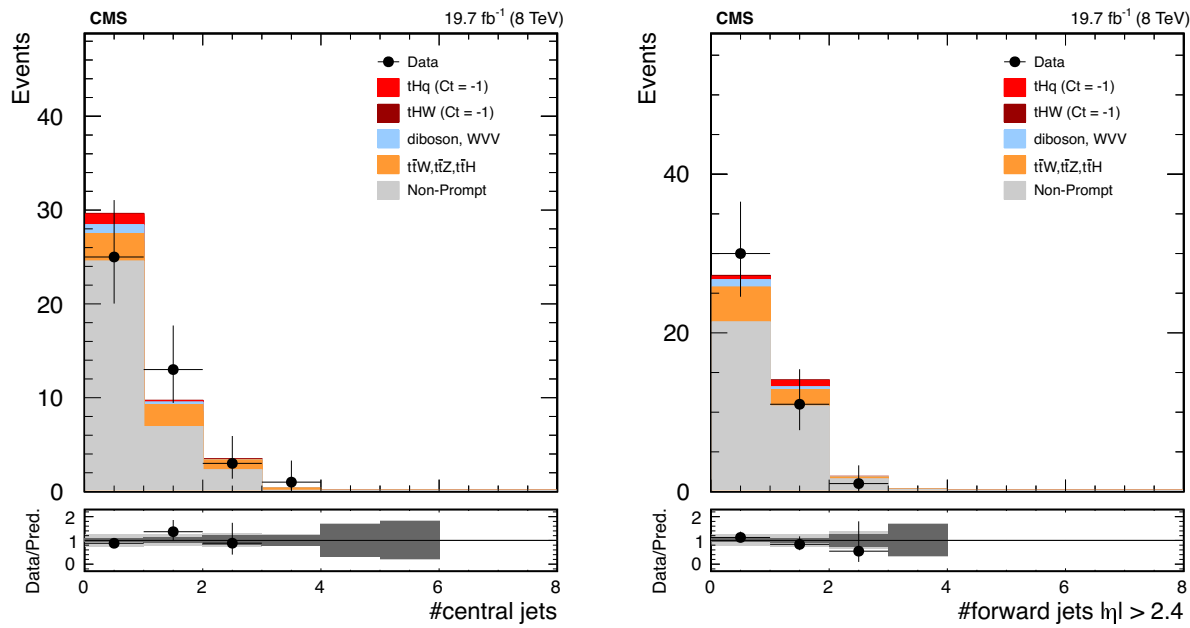

Figure 10. Sample of input variables to the final discriminant in the $\mathrm{H} \rightarrow \mathrm{WW}$ analysis. Left: distribution of the number of central jets. Right: distribution of the number of forward jets. The signal contribution is normalized to the prediction of the anomalous coupling hypothesis and stacked on top of the predicted background contributions. The bottom panels show the ratio between data and predictions, together with the statistical and systematic uncertainty bands.

discriminates well between the tHq signal and the background from non-prompt leptons, which originates mostly from t $\bar{t}$ production. Both distributions are shown in figure 10 for the events containing three charged leptons.

In the $\mathrm{H} \rightarrow \tau \tau$ analysis it is important to understand both the kinematics and the rate of the reducible backgrounds. The background estimation from leptons falsely identified as hadronically-decaying $\tau$ leptons is validated in a control region where the light-lepton 

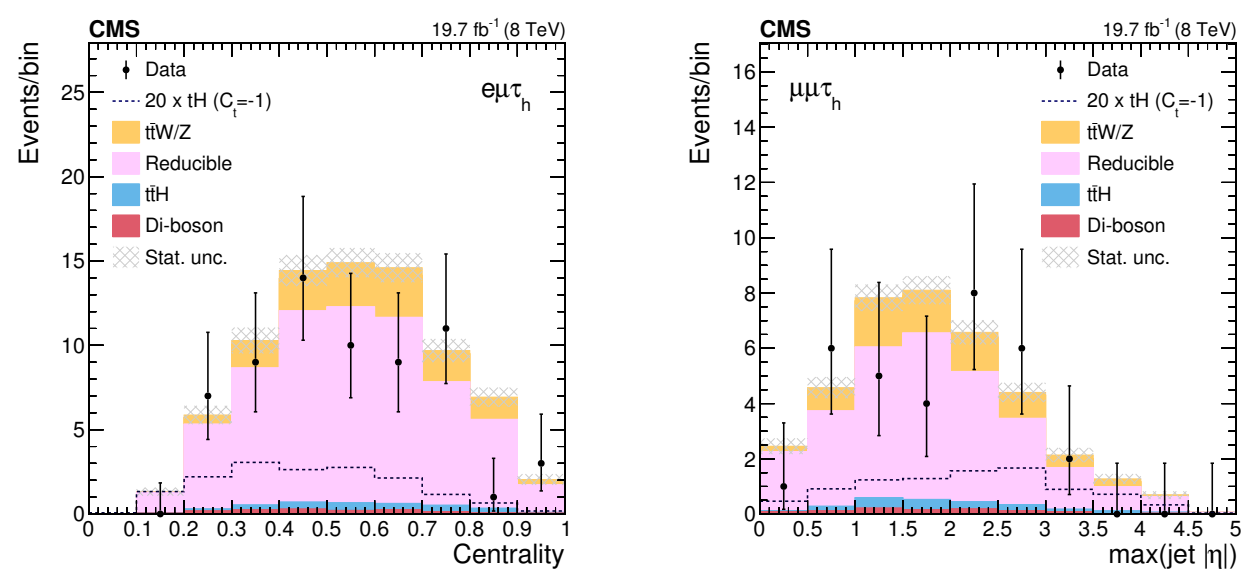

Figure 11. Sample of input variables to the final discriminant in the $H \rightarrow \tau \tau$ analysis. The distributions are shown for events failing the tight electron and muon isolation criteria. Left: distribution of the centrality for events with an electron, a muon, and a hadronically-decaying tau lepton. Right: distribution of the rapidity of the most forward jet in events with two muons and a hadronically-decaying tau lepton. The dashed line shows the expected contribution from the tHq signal $\left(C_{\mathrm{t}}=-1\right)$ case, multiplied by 20 .

same-charge requirement is maintained, but the isolation requirement on the $\tau_{\mathrm{h}}$ candidate is inverted. This region is dominated by $\mathrm{W}+$ jets and $\mathrm{t} \overline{\mathrm{t}}+$ jets backgrounds. The agreement between data and predictions in this control region is shown for the $\mathrm{e} \mu \tau_{\mathrm{h}}$ and $\mu \mu \tau_{\mathrm{h}}$ channels in figure 11. The residual differences are taken into account as systematic uncertainties in the analysis.

Open Access. This article is distributed under the terms of the Creative Commons Attribution License (CC-BY 4.0), which permits any use, distribution and reproduction in any medium, provided the original author(s) and source are credited.

\section{References}

[1] CMS collaboration, Observation of a new boson at a mass of $125 \mathrm{GeV}$ with the CMS experiment at the LHC, Phys. Lett. B 716 (2012) 30 [arXiv:1207.7235] [INSPIRE].

[2] ATLAS collaboration, Observation of a new particle in the search for the standard model Higgs boson with the ATLAS detector at the LHC, Phys. Lett. B 716 (2012) 1 [arXiv: 1207.7214] [INSPIRE].

[3] CMS collaboration, Observation of a new boson with mass near $125 \mathrm{GeV}$ in pp collisions at $\sqrt{s}=7$ and $8 \mathrm{TeV}$, JHEP 06 (2013) 081 [arXiv:1303.4571] [INSPIRE].

[4] CMS collaboration, Evidence for the direct decay of the $125 \mathrm{GeV}$ Higgs boson to fermions, Nature Phys. 10 (2014) 557 [arXiv:1401.6527] [INSPIRE].

[5] ATLAS collaboration, Evidence for the Higgs-boson Yukawa coupling to $\tau$ leptons with the ATLAS detector, JHEP 04 (2015) 117 [arXiv: 1501.04943] [INSPIRE].

[6] CDF, D0 collaboration, T. Aaltonen et al., Evidence for a particle produced in association with weak bosons and decaying to a bottom-antibottom quark pair in Higgs boson searches at the Tevatron, Phys. Rev. Lett. 109 (2012) 071804 [arXiv:1207.6436] [INSPIRE]. 
[7] CMS collaboration, Search for the standard model Higgs boson produced in association with a $W$ or a $Z$ boson and decaying to bottom quarks, Phys. Rev. D 89 (2014) 012003.

[8] CMS collaboration, Evidence for the 125 GeV Higgs boson decaying to a pair of $\tau$ leptons, JHEP 05 (2014) 104 [arXiv:1401.5041] [INSPIRE].

[9] ATLAS, CDF, CMS, D0 collaboration, First combination of Tevatron and LHC measurements of the top-quark mass, arXiv:1403.4427 [INSPIRE].

[10] CMS collaboration, Search for the associated production of the Higgs boson with a top-quark pair, JHEP 09 (2014) 087 [Erratum ibid. 10 (2014) 106] [arXiv:1408.1682] [INSPIRE].

[11] CMS collaboration, Search for a standard model Higgs boson produced in association with a top-quark pair and decaying to bottom quarks using a matrix element method, Eur. Phys. J. C 75 (2015) 251 [arXiv: 1502.02485] [INSPIRE].

[12] ATLAS collaboration, Search for $H \rightarrow \gamma \gamma$ produced in association with top quarks and constraints on the Yukawa coupling between the top quark and the Higgs boson using data taken at $7 \mathrm{TeV}$ and $8 \mathrm{TeV}$ with the ATLAS detector, Phys. Lett. B 740 (2015) 222 [arXiv:1409.3122] [INSPIRE].

[13] ATLAS collaboration, Search for the Standard Model Higgs boson produced in association with top quarks and decaying into bj in pp collisions at $\sqrt{s}=8$ TeV with the ATLAS detector, Eur. Phys. J. C 75 (2015) 349 [arXiv:1503.05066] [INSPIRE].

[14] F. Maltoni, K. Paul, T. Stelzer and S. Willenbrock, Associated production of Higgs and single top at hadron colliders, Phys. Rev. D 64 (2001) 094023 [hep-ph/0106293] [INSPIRE].

[15] M. Farina, C. Grojean, F. Maltoni, E. Salvioni and A. Thamm, Lifting degeneracies in Higgs couplings using single top production in association with a Higgs boson, JHEP 05 (2013) 022 [arXiv: 1211.3736] [INSPIRE].

[16] P. Agrawal, S. Mitra and A. Shivaji, Effect of anomalous couplings on the associated production of a single top quark and a Higgs boson at the LHC, JHEP 12 (2013) 077 [arXiv: 1211.4362] [INSPIRE].

[17] S. Biswas, E. Gabrielli and B. Mele, Single top and Higgs associated production as a probe of the Htt coupling sign at the LHC, JHEP 01 (2013) 088 [arXiv: 1211.0499] [INSPIRE].

[18] S. Biswas, E. Gabrielli, F. Margaroli and B. Mele, Direct constraints on the top-Higgs coupling from the $8 \mathrm{TeV} \mathrm{LHC} \mathrm{data,} \mathrm{JHEP} 07$ (2013) 073 [arXiv:1304.1822] [INSPIRE].

[19] J. Yue, Enhanced thj signal at the LHC with $h \rightarrow \gamma \gamma$ decay and CP-violating top-Higgs coupling, Phys. Lett. B 744 (2015) 131 [arXiv:1410.2701] [INSPIRE].

[20] A. Kobakhidze, L. Wu and J. Yue, Anomalous top-Higgs couplings and top polarisation in single top and Higgs associated production at the LHC, JHEP 10 (2014) 100 [arXiv: 1406.1961] [INSPIRE].

[21] F. Demartin, F. Maltoni, K. Mawatari and M. Zaro, Higgs production in association with a single top quark at the LHC, Eur. Phys. J. C 75 (2015) 267 [arXiv:1504.00611] [INSPIRE].

[22] J.A. Aguilar-Saavedra, R. Benbrik, S. Heinemeyer and M. Pérez-Victoria, Handbook of vectorlike quarks: mixing and single production, Phys. Rev. D 88 (2013) 094010 [arXiv: 1306.0572] [INSPIRE].

[23] A. Greljo, J.F. Kamenik and J. Kopp, Disentangling flavor violation in the top-Higgs sector at the LHC, JHEP 07 (2014) 046 [arXiv: 1404.1278] [INSPIRE]. 
[24] J. Ellis and T. You, Updated global analysis of Higgs couplings, JHEP 06 (2013) 103 [arXiv: 1303.3879] [INSPIRE].

[25] CMS collaboration, The CMS experiment at the CERN LHC, 2008 JINST 3 S08004 [INSPIRE].

[26] CMS collaboration, Particle-flow event reconstruction in CMS and performance for jets, taus and MET, CMS-PAS-PFT-09-001 (2009).

[27] CMS collaboration, Commissioning of the particle-flow event reconstruction with the first LHC collisions recorded in the CMS detector, CMS-PAS-PFT-10-001 (2010).

[28] CMS collaboration, Observation of the diphoton decay of the Higgs boson and measurement of its properties, Eur. Phys. J. C 74 (2014) 3076 [arXiv:1407.0558] [INSPIRE].

[29] CMS collaboration, Performance of photon reconstruction and identification with the CMS detector in proton-proton collisions at $\sqrt{s}=8 \mathrm{TeV}, 2015$ JINST $10 \mathrm{P} 08010$ [arXiv: 1502.02702] [INSPIRE].

[30] CMS collaboration, Performance of electron reconstruction and selection with the CMS detector in proton-proton collisions at $\sqrt{s}=8 \mathrm{TeV}, 2015$ JINST $10 \mathrm{P} 06005$ [arXiv: 1502.02701] [INSPIRE].

[31] CMS collaboration, Performance of CMS muon reconstruction in pp collision events at $\sqrt{s}=7 \mathrm{TeV}, 2012$ JINST 7 P10002 [arXiv:1206.4071] [INSPIRE]

[32] CMS collaboration, Particle-flow commissioning with muons and electrons from $J / \Psi$ and $W$ events at $7 \mathrm{TeV}$, CMS-PAS-PFT-10-003 (2010).

[33] M. Cacciari, G.P. Salam and G. Soyez, The anti- $k_{t}$ jet clustering algorithm, JHEP 04 (2008) 063 [arXiv: 0802.1189] [INSPIRE].

[34] M. Cacciari, G.P. Salam and G. Soyez, FastJet user manual, Eur. Phys. J. C 72 (2012) 1896 [arXiv: 1111.6097] [INSPIRE].

[35] M. Cacciari, G.P. Salam and G. Soyez, The catchment area of jets, JHEP 04 (2008) 005 [arXiv: 0802.1188] [INSPIRE].

[36] CMS collaboration, Determination of jet energy calibration and transverse momentum resolution in CMS, 2011 JINST 6 P11002 [arXiv:1107.4277] [INSPIRE].

[37] CMS collaboration, Performance of $\tau$-lepton reconstruction and identification in CMS, 2012 JINST 7 P01001.

[38] CMS collaboration, Performance of b tagging at $\sqrt{s}=8 \mathrm{TeV}$ in multijet, ttbar and boosted topology events, CMS-PAS-BTV-13-001 (2013).

[39] CMS collaboration, Identification of b-quark jets with the CMS experiment, 2013 JINST 8 P04013 [arXiv: 1211.4462] [INSPIRE].

[40] J. Alwall, M. Herquet, F. Maltoni, O. Mattelaer and T. Stelzer, MadGraph 5: going beyond, JHEP 06 (2011) 128 [arXiv:1106.0522] [INSPIRE].

[41] T. Sjöstrand, S. Mrenna and P.Z. Skands, PYTHIA 6.4 physics and manual, JHEP 05 (2006) 026 [hep-ph/0603175] [INSPIRE].

[42] H.-L. Lai et al., Uncertainty induced by QCD coupling in the CTEQ global analysis of parton distributions, Phys. Rev. D 82 (2010) 054021 [arXiv:1004.4624] [INSPIRE]. 
[43] S. Frixione, P. Nason and C. Oleari, Matching NLO QCD computations with parton shower simulations: the POWHEG method, JHEP 11 (2007) 070 [arXiv: 0709.2092] [INSPIRE].

[44] S. Alioli, P. Nason, C. Oleari and E. Re, A general framework for implementing NLO calculations in shower Monte Carlo programs: the POWHEG BOX, JHEP 06 (2010) 043 [arXiv: 1002.2581] [INSPIRE].

[45] GEANT4 collaboration, S. Agostinelli et al., GEANT4 - a simulation toolkit, Nucl. Instrum. Meth. A 506 (2003) 250 [INSPIRE].

[46] F. Micheli, Searching for new physics in the Higgs sector: the $H \rightarrow \gamma \gamma$ channel at CMS, Ph.D. thesis, Sapienza University, Rome, Italy (2014).

[47] A. Popov, Search for anomalous Higgs boson production in association with single top quarks using the CMS detector, Ph.D. thesis, Université catholique de Louvain, Louvain, Belgium (2015), CERN-THESIS-2015-177.

[48] A. Höcker et al., TMVA - Toolkit for Multivariate Data Analysis, PoS (ACAT) 040 [physics/0703039] [INSPIRE].

[49] CMS collaboration, Measurements of inclusive $W$ and $Z$ cross sections in pp collisions at $\sqrt{s}=7 \mathrm{TeV}, J H E P 01$ (2011) 080 [arXiv:1012.2466] [INSPIRE].

[50] CMS collaboration, Energy calibration and resolution of the CMS electromagnetic calorimeter in pp collisions at $\sqrt{s}=7$ TeV, 2013 JINST 8 P09009 [arXiv:1306.2016] [INSPIRE].

[51] S. Alekhin et al., The PDF4LHC working group interim report, arXiv:1101.0536 [INSPIRE].

[52] M. Botje et al., The PDF4LHC working group interim recommendations, arXiv:1101.0538 [INSPIRE].

[53] CMS collaboration, Measurement of the differential cross section for top quark pair production in pp collisions at $\sqrt{s}=8 \mathrm{TeV}$, Eur. Phys. J. C 75 (2015) 542 [arXiv: 1505.04480] [INSPIRE].

[54] CMS collaboration, CMS luminosity based on pixel cluster counting - Summer 2013 update, CMS-PAS-LUM-13-001 (2013).

[55] A.L. Read, Presentation of search results: the $C L_{s}$ technique, J. Phys. G 28 (2002) 2693 [INSPIRE].

[56] T. Junk, Confidence level computation for combining searches with small statistics, Nucl. Instrum. Meth. A 434 (1999) 435 [hep-ex/9902006] [INSPIRE].

[57] V. Hirschi, R. Frederix, S. Frixione, M.V. Garzelli, F. Maltoni and R. Pittau, Automation of one-loop QCD corrections, JHEP 05 (2011) 044 [arXiv:1103.0621] [INSPIRE]. 


\section{The CMS collaboration}

\section{Yerevan Physics Institute, Yerevan, Armenia}

V. Khachatryan, A.M. Sirunyan, A. Tumasyan

Institut für Hochenergiephysik der OeAW, Wien, Austria

W. Adam, E. Asilar, T. Bergauer, J. Brandstetter, E. Brondolin, M. Dragicevic, J. Erö, M. Flechl, M. Friedl, R. Frühwirth'1 ${ }^{1}$, V.M. Ghete, C. Hartl, N. Hörmann, J. Hrubec, M. Jeitler ${ }^{1}$, V. Knünz, A. König, M. Krammer ${ }^{1}$, I. Krätschmer, D. Liko, T. Matsushita, I. Mikulec, D. Rabady ${ }^{2}$, B. Rahbaran, H. Rohringer, J. Schieck ${ }^{1}$, R. Schöfbeck, J. Strauss, W. Treberer-Treberspurg, W. Waltenberger, C.-E. Wulz ${ }^{1}$

National Centre for Particle and High Energy Physics, Minsk, Belarus

V. Mossolov, N. Shumeiko, J. Suarez Gonzalez

Universiteit Antwerpen, Antwerpen, Belgium

S. Alderweireldt, T. Cornelis, E.A. De Wolf, X. Janssen, A. Knutsson, J. Lauwers, S. Luyckx, M. Van De Klundert, H. Van Haevermaet, P. Van Mechelen, N. Van Remortel, A. Van Spilbeeck

\section{Vrije Universiteit Brussel, Brussel, Belgium}

S. Abu Zeid, F. Blekman, J. D'Hondt, N. Daci, I. De Bruyn, K. Deroover, N. Heracleous, J. Keaveney, S. Lowette, L. Moreels, A. Olbrechts, Q. Python, D. Strom, S. Tavernier, W. Van Doninck, P. Van Mulders, G.P. Van Onsem, I. Van Parijs

\section{Université Libre de Bruxelles, Bruxelles, Belgium}

P. Barria, H. Brun, C. Caillol, B. Clerbaux, G. De Lentdecker, G. Fasanella, L. Favart, A. Grebenyuk, G. Karapostoli, T. Lenzi, A. Léonard, T. Maerschalk, A. Marinov, L. Perniè, A. Randle-conde, T. Reis, T. Seva, C. Vander Velde, P. Vanlaer, R. Yonamine, F. Zenoni, F. Zhang ${ }^{3}$

\section{Ghent University, Ghent, Belgium}

K. Beernaert, L. Benucci, A. Cimmino, S. Crucy, D. Dobur, A. Fagot, G. Garcia, M. Gul, J. Mccartin, A.A. Ocampo Rios, D. Poyraz, D. Ryckbosch, S. Salva, M. Sigamani, N. Strobbe, M. Tytgat, W. Van Driessche, E. Yazgan, N. Zaganidis

\section{Université Catholique de Louvain, Louvain-la-Neuve, Belgium}

S. Basegmez, C. Beluffi ${ }^{4}$, O. Bondu, S. Brochet, G. Bruno, A. Caudron, L. Ceard, G.G. Da Silveira, C. Delaere, D. Favart, L. Forthomme, A. Giammanco ${ }^{5}$, J. Hollar, A. Jafari, P. Jez, M. Komm, V. Lemaitre, A. Mertens, M. Musich, C. Nuttens, L. Perrini, A. Pin, K. Piotrzkowski, A. Popov ${ }^{6}$, L. Quertenmont, M. Selvaggi, M. Vidal Marono

\section{Université de Mons, Mons, Belgium}

N. Beliy, G.H. Hammad

\section{Centro Brasileiro de Pesquisas Fisicas, Rio de Janeiro, Brazil}

W.L. Aldá Júnior, F.L. Alves, G.A. Alves, L. Brito, M. Correa Martins Junior, M. Hamer, C. Hensel, C. Mora Herrera, A. Moraes, M.E. Pol, P. Rebello Teles 
Universidade do Estado do Rio de Janeiro, Rio de Janeiro, Brazil

E. Belchior Batista Das Chagas, W. Carvalho, J. Chinellato ${ }^{7}$, A. Custódio, E.M. Da Costa, D. De Jesus Damiao, C. De Oliveira Martins, S. Fonseca De Souza, L.M. Huertas Guativa, H. Malbouisson, D. Matos Figueiredo, L. Mundim, H. Nogima, W.L. Prado Da Silva, A. Santoro, A. Sznajder, E.J. Tonelli Manganote ${ }^{7}$, A. Vilela Pereira

Universidade Estadual Paulista $^{a}$, Universidade Federal do ABC ${ }^{b}$, São Paulo, Brazil

S. Ahuja ${ }^{a}$, C.A. Bernardes ${ }^{b}$, A. De Souza Santos ${ }^{b}$, S. Dogra $^{a}$, T.R. Fernandez Perez Tomei ${ }^{a}$, E.M. Gregores ${ }^{b}$, P.G. Mercadante ${ }^{b}$, C.S. Moon ${ }^{a, 8}$, S.F. Novaes ${ }^{a}$, Sandra S. Padula ${ }^{a}$, D. Romero Abad, J.C. Ruiz Vargas

Institute for Nuclear Research and Nuclear Energy, Sofia, Bulgaria

A. Aleksandrov, R. Hadjiiska, P. Iaydjiev, M. Rodozov, S. Stoykova, G. Sultanov, M. Vutova

University of Sofia, Sofia, Bulgaria

A. Dimitrov, I. Glushkov, L. Litov, B. Pavlov, P. Petkov

Institute of High Energy Physics, Beijing, China

M. Ahmad, J.G. Bian, G.M. Chen, H.S. Chen, M. Chen, T. Cheng, R. Du, C.H. Jiang, R. Plestina ${ }^{9}$, F. Romeo, S.M. Shaheen, J. Tao, C. Wang, Z. Wang, H. Zhang

State Key Laboratory of Nuclear Physics and Technology, Peking University, Beijing, China

C. Asawatangtrakuldee, Y. Ban, Q. Li, S. Liu, Y. Mao, S.J. Qian, D. Wang, Z. Xu

Universidad de Los Andes, Bogota, Colombia

C. Avila, A. Cabrera, L.F. Chaparro Sierra, C. Florez, J.P. Gomez, B. Gomez Moreno, J.C. Sanabria

University of Split, Faculty of Electrical Engineering, Mechanical Engineering and Naval Architecture, Split, Croatia

N. Godinovic, D. Lelas, I. Puljak, P.M. Ribeiro Cipriano

University of Split, Faculty of Science, Split, Croatia

Z. Antunovic, M. Kovac

Institute Rudjer Boskovic, Zagreb, Croatia

V. Brigljevic, K. Kadija, J. Luetic, S. Micanovic, L. Sudic

University of Cyprus, Nicosia, Cyprus

A. Attikis, G. Mavromanolakis, J. Mousa, C. Nicolaou, F. Ptochos, P.A. Razis, H. Rykaczewski

Charles University, Prague, Czech Republic

M. Bodlak, M. Finger ${ }^{10}$, M. Finger Jr. ${ }^{10}$ 
Academy of Scientific Research and Technology of the Arab Republic of Egypt, Egyptian Network of High Energy Physics, Cairo, Egypt

Y. Assran ${ }^{11}$, M. El Sawy ${ }^{12,13}$, S. Elgammal ${ }^{13}$, A. Ellithi Kamel ${ }^{14,14}$, M.A. Mahmoud ${ }^{15,15}$

National Institute of Chemical Physics and Biophysics, Tallinn, Estonia

B. Calpas, M. Kadastik, M. Murumaa, M. Raidal, A. Tiko, C. Veelken

Department of Physics, University of Helsinki, Helsinki, Finland

P. Eerola, J. Pekkanen, M. Voutilainen

Helsinki Institute of Physics, Helsinki, Finland

J. Härkönen, V. Karimäki, R. Kinnunen, T. Lampén, K. Lassila-Perini, S. Lehti, T. Lindén, P. Luukka, T. Mäenpää, T. Peltola, E. Tuominen, J. Tuominiemi, E. Tuovinen, L. Wendland

Lappeenranta University of Technology, Lappeenranta, Finland

J. Talvitie, T. Tuuva

DSM/IRFU, CEA/Saclay, Gif-sur-Yvette, France

M. Besancon, F. Couderc, M. Dejardin, D. Denegri, B. Fabbro, J.L. Faure, C. Favaro, F. Ferri, S. Ganjour, A. Givernaud, P. Gras, G. Hamel de Monchenault, P. Jarry, E. Locci, M. Machet, J. Malcles, J. Rander, A. Rosowsky, M. Titov, A. Zghiche

Laboratoire Leprince-Ringuet, Ecole Polytechnique, IN2P3-CNRS, Palaiseau, France

I. Antropov, S. Baffioni, F. Beaudette, P. Busson, L. Cadamuro, E. Chapon, C. Charlot, T. Dahms, O. Davignon, N. Filipovic, A. Florent, R. Granier de Cassagnac, S. Lisniak, L. Mastrolorenzo, P. Miné, I.N. Naranjo, M. Nguyen, C. Ochando, G. Ortona, P. Paganini, P. Pigard, S. Regnard, R. Salerno, J.B. Sauvan, Y. Sirois, T. Strebler, Y. Yilmaz, A. Zabi

Institut Pluridisciplinaire Hubert Curien, Université de Strasbourg, Université de Haute Alsace Mulhouse, CNRS/IN2P3, Strasbourg, France

J.-L. Agram ${ }^{16}$, J. Andrea, A. Aubin, D. Bloch, J.-M. Brom, M. Buttignol, E.C. Chabert, N. Chanon, C. Collard, E. Conte ${ }^{16}$, X. Coubez, J.-C. Fontaine ${ }^{16}$, D. Gelé, U. Goerlach, C. Goetzmann, A.-C. Le Bihan, J.A. Merlin², K. Skovpen, P. Van Hove

Centre de Calcul de l'Institut National de Physique Nucleaire et de Physique des Particules, CNRS/IN2P3, Villeurbanne, France

S. Gadrat

Université de Lyon, Université Claude Bernard Lyon 1, CNRS-IN2P3, Institut de Physique Nucléaire de Lyon, Villeurbanne, France

S. Beauceron, C. Bernet, G. Boudoul, E. Bouvier, C.A. Carrillo Montoya, R. Chierici, D. Contardo, B. Courbon, P. Depasse, H. El Mamouni, J. Fan, J. Fay, S. Gascon, M. Gouzevitch, B. Ille, F. Lagarde, I.B. Laktineh, M. Lethuillier, L. Mirabito, A.L. Pequegnot, S. Perries, J.D. Ruiz Alvarez, D. Sabes, L. Sgandurra, V. Sordini, M. Vander Donckt, P. Verdier, S. Viret 
Georgian Technical University, Tbilisi, Georgia

T. Toriashvili ${ }^{17}$

\section{Tbilisi State University, Tbilisi, Georgia}

Z. Tsamalaidze ${ }^{10}$

RWTH Aachen University, I. Physikalisches Institut, Aachen, Germany

C. Autermann, S. Beranek, M. Edelhoff, L. Feld, A. Heister, M.K. Kiesel, K. Klein, M. Lipinski, A. Ostapchuk, M. Preuten, F. Raupach, S. Schael, J.F. Schulte, T. Verlage, H. Weber, B. Wittmer, V. Zhukov ${ }^{6}$

RWTH Aachen University, III. Physikalisches Institut A, Aachen, Germany M. Ata, M. Brodski, E. Dietz-Laursonn, D. Duchardt, M. Endres, M. Erdmann, S. Erdweg, T. Esch, R. Fischer, A. Güth, T. Hebbeker, C. Heidemann, K. Hoepfner, D. Klingebiel, S. Knutzen, P. Kreuzer, M. Merschmeyer, A. Meyer, P. Millet, M. Olschewski, K. Padeken, P. Papacz, T. Pook, M. Radziej, H. Reithler, M. Rieger, F. Scheuch, L. Sonnenschein, D. Teyssier, S. Thüer

RWTH Aachen University, III. Physikalisches Institut B, Aachen, Germany

V. Cherepanov, Y. Erdogan, G. Flügge, H. Geenen, M. Geisler, F. Hoehle, B. Kargoll, T. Kress, Y. Kuessel, A. Künsken, J. Lingemann² ${ }^{2}$ A. Nehrkorn, A. Nowack, I.M. Nugent, C. Pistone, O. Pooth, A. Stahl

\section{Deutsches Elektronen-Synchrotron, Hamburg, Germany}

M. Aldaya Martin, I. Asin, N. Bartosik, O. Behnke, U. Behrens, A.J. Bell, K. Borras ${ }^{18}$, A. Burgmeier, A. Cakir, A. Campbell, S. Choudhury, F. Costanza, C. Diez Pardos, G. Dolinska, S. Dooling, T. Dorland, G. Eckerlin, D. Eckstein, T. Eichhorn, G. Flucke, E. Gallo ${ }^{19}$, J. Garay Garcia, A. Geiser, A. Gizhko, P. Gunnellini, J. Hauk, M. Hempel ${ }^{20}$, H. Jung, A. Kalogeropoulos, O. Karacheban ${ }^{20}$, M. Kasemann, P. Katsas, J. Kieseler, C. Kleinwort, I. Korol, W. Lange, J. Leonard, K. Lipka, A. Lobanov, W. Lohmann ${ }^{20}$, R. Mankel, I. Marfin ${ }^{20}$, I.-A. Melzer-Pellmann, A.B. Meyer, G. Mittag, J. Mnich, A. Mussgiller, S. Naumann-Emme, A. Nayak, E. Ntomari, H. Perrey, D. Pitzl, R. Placakyte, A. Raspereza, B. Roland, M.Ö. Sahin, P. Saxena, T. Schoerner-Sadenius, M. Schröder, C. Seitz, S. Spannagel, K.D. Trippkewitz, R. Walsh, C. Wissing

\section{University of Hamburg, Hamburg, Germany}

V. Blobel, M. Centis Vignali, A.R. Draeger, J. Erfle, E. Garutti, K. Goebel, D. Gonzalez, M. Görner, J. Haller, M. Hoffmann, R.S. Höing, A. Junkes, R. Klanner, R. Kogler, T. Lapsien, T. Lenz, I. Marchesini, D. Marconi, M. Meyer, D. Nowatschin, J. Ott, F. Pantaleo ${ }^{2}$, T. Peiffer, A. Perieanu, N. Pietsch, J. Poehlsen, D. Rathjens, C. Sander, H. Schettler, P. Schleper, E. Schlieckau, A. Schmidt, J. Schwandt, M. Seidel, V. Sola, H. Stadie, G. Steinbrück, H. Tholen, D. Troendle, E. Usai, L. Vanelderen, A. Vanhoefer, B. Vormwald

\section{Institut für Experimentelle Kernphysik, Karlsruhe, Germany}

M. Akbiyik, C. Barth, C. Baus, J. Berger, C. Böser, E. Butz, T. Chwalek, F. Colombo, W. De Boer, A. Descroix, A. Dierlamm, N. Faltermann, S. Fink, F. Frensch, M. Giffels, 
A. Gilbert, F. Hartmann ${ }^{2}$, S.M. Heindl, U. Husemann, I. Katkov ${ }^{6}$, A. Kornmayer ${ }^{2}$, P. Lobelle Pardo, B. Maier, H. Mildner, M.U. Mozer, T. Müller, Th. Müller, M. Plagge, G. Quast, K. Rabbertz, S. Röcker, F. Roscher, H.J. Simonis, F.M. Stober, R. Ulrich, J. Wagner-Kuhr, S. Wayand, M. Weber, T. Weiler, C. Wöhrmann, R. Wolf

Institute of Nuclear and Particle Physics (INPP), NCSR Demokritos, Aghia Paraskevi, Greece

G. Anagnostou, G. Daskalakis, T. Geralis, V.A. Giakoumopoulou, A. Kyriakis, D. Loukas, A. Psallidas, I. Topsis-Giotis

University of Athens, Athens, Greece

A. Agapitos, S. Kesisoglou, A. Panagiotou, N. Saoulidou, E. Tziaferi

University of Ioánnina, Ioánnina, Greece

I. Evangelou, G. Flouris, C. Foudas, P. Kokkas, N. Loukas, N. Manthos, I. Papadopoulos, E. Paradas, J. Strologas

Wigner Research Centre for Physics, Budapest, Hungary

G. Bencze, C. Hajdu, A. Hazi, P. Hidas, D. Horvath ${ }^{21}$, F. Sikler, V. Veszpremi, G. Vesztergombi ${ }^{22}$, A.J. Zsigmond

Institute of Nuclear Research ATOMKI, Debrecen, Hungary

N. Beni, S. Czellar, J. Karancsi ${ }^{23}$, J. Molnar, Z. Szillasi

University of Debrecen, Debrecen, Hungary

M. Bartók ${ }^{24}$, A. Makovec, P. Raics, Z.L. Trocsanyi, B. Ujvari

National Institute of Science Education and Research, Bhubaneswar, India

P. Mal, K. Mandal, D.K. Sahoo, N. Sahoo, S.K. Swain

Panjab University, Chandigarh, India

S. Bansal, S.B. Beri, V. Bhatnagar, R. Chawla, R. Gupta, U.Bhawandeep, A.K. Kalsi, A. Kaur, M. Kaur, R. Kumar, A. Mehta, M. Mittal, J.B. Singh, G. Walia

University of Delhi, Delhi, India

Ashok Kumar, A. Bhardwaj, B.C. Choudhary, R.B. Garg, A. Kumar, S. Malhotra, M. Naimuddin, N. Nishu, K. Ranjan, R. Sharma, V. Sharma

Saha Institute of Nuclear Physics, Kolkata, India

S. Bhattacharya, K. Chatterjee, S. Dey, S. Dutta, Sa. Jain, N. Majumdar, A. Modak, K. Mondal, S. Mukherjee, S. Mukhopadhyay, A. Roy, D. Roy, S. Roy Chowdhury, S. Sarkar, M. Sharan

Bhabha Atomic Research Centre, Mumbai, India

A. Abdulsalam, R. Chudasama, D. Dutta, V. Jha, V. Kumar, A.K. Mohanty ${ }^{2}$, L.M. Pant, P. Shukla, A. Topkar

Tata Institute of Fundamental Research, Mumbai, India

T. Aziz, S. Banerjee, S. Bhowmik ${ }^{25}$, R.M. Chatterjee, R.K. Dewanjee, S. Dugad, S. Ganguly, S. Ghosh, M. Guchait, A. Gurtu ${ }^{26}$, G. Kole, S. Kumar, B. Mahakud, M. Maity ${ }^{25}$, 
G. Majumder, K. Mazumdar, S. Mitra, G.B. Mohanty, B. Parida, T. Sarkar ${ }^{25}$, N. Sur, B. Sutar, N. Wickramage ${ }^{27}$

Indian Institute of Science Education and Research (IISER), Pune, India

S. Chauhan, S. Dube, S. Sharma

Institute for Research in Fundamental Sciences (IPM), Tehran, Iran

H. Bakhshiansohi, H. Behnamian, S.M. Etesami28, A. Fahim²9, R. Goldouzian, M. Khakzad, M. Mohammadi Najafabadi, M. Naseri, S. Paktinat Mehdiabadi, F. Rezaei Hosseinabadi, B. Safarzadeh ${ }^{30}$, M. Zeinali

University College Dublin, Dublin, Ireland

M. Felcini, M. Grunewald

INFN Sezione di Bari ${ }^{a}$, Università di Bari ${ }^{b}$, Politecnico di Bari ${ }^{c}$, Bari, Italy M. Abbrescia ${ }^{a, b}$, C. Calabria ${ }^{a, b}$, C. Caputo ${ }^{a, b}$, A. Colaleo $^{a}$, D. Creanza ${ }^{a, c}$, L. Cristella $^{a, b}$, N. De Filippis ${ }^{a, c}$, M. De Palma ${ }^{a, b}$, L. Fiore ${ }^{a}$, G. Iaselli ${ }^{a, c}$, G. Maggi ${ }^{a, c}$, M. Maggi $^{a}$, G. Miniello ${ }^{a, b}$, S. My ${ }^{a, c}$, S. Nuzzo ${ }^{a, b}$, A. Pompili ${ }^{a, b}$, G. Pugliese ${ }^{a, c}$, R. Radogna ${ }^{a, b}$,

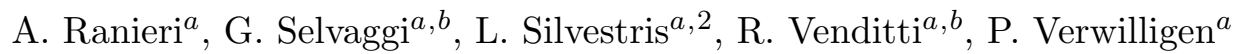

INFN Sezione di Bologna ${ }^{a}$, Università di Bologna ${ }^{b}$, Bologna, Italy

G. Abbiendi ${ }^{a}$, C. Battilana ${ }^{2}$, A.C. Benvenuti ${ }^{a}$, D. Bonacorsi ${ }^{a}, b$, S. Braibant-Giacomelli ${ }^{a, b}$, L. Brigliadori ${ }^{a, b}$, R. Campanini ${ }^{a, b}$, P. Capiluppi ${ }^{a, b}$, A. Castro ${ }^{a, b}$, F.R. Cavallo ${ }^{a}$, S.S. Chhibra ${ }^{a, b}$, G. Codispoti ${ }^{a, b}$, M. Cuffiani ${ }^{a, b}$, G.M. Dallavalle ${ }^{a}$, F. Fabbri ${ }^{a}$, A. Fanfani $^{a, b}{ }^{,}$ D. Fasanella ${ }^{a, b}$, P. Giacomelli ${ }^{a}$, C. Grandi ${ }^{a}$, L. Guiducci ${ }^{a}, b$, S. Marcellini ${ }^{a}$, G. Masetti ${ }^{a}$,

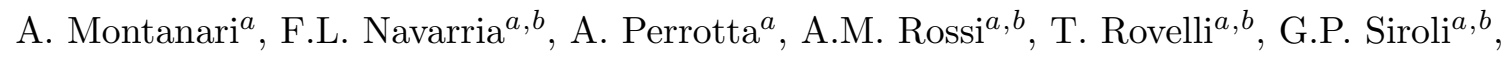
N. Tosi ${ }^{a, b}$, R. Travaglini ${ }^{a, b}$

INFN Sezione di Catania ${ }^{a}$, Università di Catania ${ }^{b}, \operatorname{CSFNSM}^{c}$, Catania, Italy

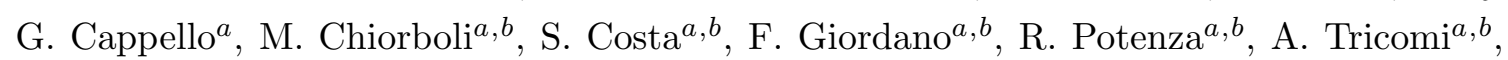
C. Tuve ${ }^{a, b}$

INFN Sezione di Firenze ${ }^{a}$, Università di Firenze ${ }^{b}$, Firenze, Italy

G. Barbagli ${ }^{a}$, V. Ciulli ${ }^{a}, b$, C. Civinini ${ }^{a}$, R. D’Alessandro ${ }^{a, b}$, E. Focardi $^{a, b}$, S. Gonzi $^{a, b}$, V. Gori ${ }^{a, b}$, P. Lenzi ${ }^{a, b}$, M. Meschini ${ }^{a}$, S. Paoletti ${ }^{a}$, G. Sguazzoni ${ }^{a}$, A. Tropiano ${ }^{a, b}$, L. Viliani ${ }^{a, b, 2}$

INFN Laboratori Nazionali di Frascati, Frascati, Italy

L. Benussi, S. Bianco, F. Fabbri, D. Piccolo, F. Primavera

INFN Sezione di Genova ${ }^{a}$, Università di Genova ${ }^{b}$, Genova, Italy

V. Calvelli ${ }^{a, b}$, F. Ferro ${ }^{a}$, M. Lo Vetere ${ }^{a, b}$, M.R. Monge ${ }^{a, b}$, E. Robutti $^{a}$, S. Tosi $^{a, b}$

INFN Sezione di Milano-Bicocca ${ }^{a}$, Università di Milano-Bicocca ${ }^{b}$, Milano, Italy

L. Brianza, M.E. Dinardo ${ }^{a, b}$, S. Fiorendi ${ }^{a, b}$, S. Gennai ${ }^{a}$, R. Gerosa ${ }^{a, b}$, A. Ghezzi ${ }^{a, b}$, P. Govoni ${ }^{a, b}$, S. Malvezzi ${ }^{a}$, R.A. Manzoni ${ }^{a, b}$, B. Marzocchi ${ }^{a, b, 2}$, D. Menasce ${ }^{a}$, L. Moroni ${ }^{a}$, M. Paganoni ${ }^{a, b}$, D. Pedrini ${ }^{a}$, S. Ragazzi ${ }^{a}, b$, N. Redaelli ${ }^{a}$, T. Tabarelli de Fatis ${ }^{a, b}$ 
INFN Sezione di Napoli ${ }^{a}$, Università di Napoli 'Federico II' ${ }^{b}$, Napoli, Italy, Università della Basilicata ${ }^{c}$, Potenza, Italy, Università G. Marconi ${ }^{d}$, Roma, Italy

S. Buontempo ${ }^{a}$, N. Cavallo $^{a, c}$, S. Di Guida ${ }^{a, d, 2}$, M. Esposito ${ }^{a, b}$, F. Fabozzi ${ }^{a, c}$, A.O.M. Iorio ${ }^{a, b}$, G. $\operatorname{Lanza}^{a}$, L. Lista ${ }^{a}$, S. Meola ${ }^{a, d, 2}$, M. Merola ${ }^{a}$, P. Paolucci ${ }^{a, 2}$, C. Sciacca ${ }^{a, b}$, F. Thyssen

INFN Sezione di Padova ${ }^{a}$, Università di Padova ${ }^{b}$, Padova, Italy, Università di Trento ${ }^{c}$, Trento, Italy

P. $\operatorname{Azzi}^{a, 2}$, N. Bacchetta ${ }^{a}$, L. Benato ${ }^{a, b}$, D. Bisello ${ }^{a, b}$, A. Boletti ${ }^{a, b}$, A. Branca ${ }^{a, b}$,

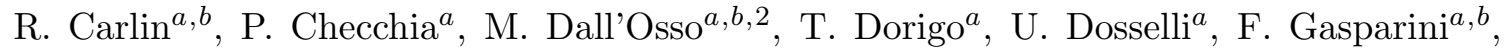
U. Gasparini ${ }^{a, b}$, A. Gozzelino ${ }^{a}$, K. Kanishchev ${ }^{a, c}$, S. Lacaprara ${ }^{a}$, M. Margoni ${ }^{a, b}$, A.T. Meneguzzo ${ }^{a, b}$, J. Pazzini ${ }^{a, b}$, N. Pozzobon ${ }^{a, b}$, P. Ronchese ${ }^{a, b}$, F. Simonetto ${ }^{a, b}$, E. Torassa ${ }^{a}$, M. Tosi ${ }^{a, b}$, S. Ventura ${ }^{a}$, M. Zanetti, P. Zotto ${ }^{a, b}$, A. Zucchetta ${ }^{a, b, 2}$, G. Zumerle ${ }^{a, b}$

INFN Sezione di Pavia ${ }^{a}$, Università di Pavia ${ }^{b}$, Pavia, Italy

A. Braghieri ${ }^{a}$, A. Magnani ${ }^{a}$, P. Montagna ${ }^{a, b}$, S.P. Ratti ${ }^{a, b}$, V. $\operatorname{Re}^{a}$, C. $\operatorname{Riccardi}^{a}{ }^{b}$,

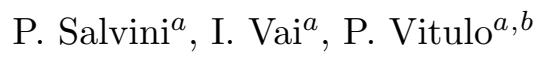

INFN Sezione di Perugia ${ }^{a}$, Università di Perugia ${ }^{b}$, Perugia, Italy

L. Alunni Solestizi ${ }^{a, b}$, M. Biasini ${ }^{a, b}$, G.M. Bilei ${ }^{a}$, D. Ciangottini ${ }^{a, b, 2}$, L. Fanò ${ }^{a, b}$, P. Lariccia ${ }^{a}, b$, G. Mantovani ${ }^{a, b}$, M. Menichelli ${ }^{a}$, A. Saha ${ }^{a}$, A. Santocchia ${ }^{a, b}$, A. Spiezia ${ }^{a, b}$

INFN Sezione di Pisa ${ }^{a}$, Università di Pisa ${ }^{b}$, Scuola Normale Superiore di Pisa ${ }^{c}$, Pisa, Italy

K. Androsov ${ }^{a, 31}$, P. Azzurri ${ }^{a}$, G. Bagliesi ${ }^{a}$, J. Bernardini ${ }^{a}$, T. Boccali ${ }^{a}$, R. Castaldi $^{a}$, M.A. Ciocci $^{a, 31}$, R. Dell'Orso ${ }^{a}$, S. Donato ${ }^{a, c, 2}$, G. Fedi, L. Foà ${ }^{a, c \dagger}$, A. Giassi ${ }^{a}$,

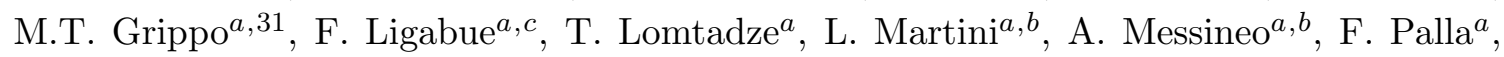

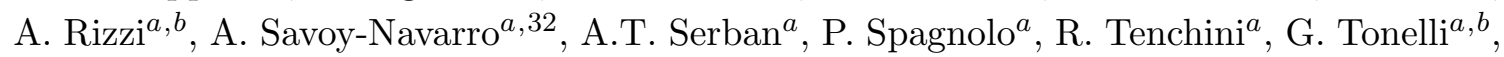
A. Venturi ${ }^{a}$, P.G. Verdini ${ }^{a}$

INFN Sezione di Roma ${ }^{a}$, Università di Roma ${ }^{b}$, Roma, Italy

L. Barone $e^{a, b}$, F. Cavallari ${ }^{a}$, G. D'imperio ${ }^{a, b, 2}$, D. Del Re ${ }^{a, b}$, M. Diemoz ${ }^{a}$, S. Gellia ${ }^{a, b}$, C. Jorda ${ }^{a}$, E. Longo ${ }^{a, b}$, F. Margaroli ${ }^{a}, b$, P. Meridiani ${ }^{a}$, G. Organtini ${ }^{a}, b$, R. Paramatti ${ }^{a}$,

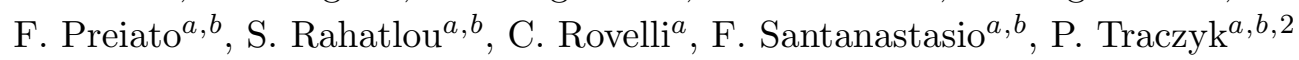

INFN Sezione di Torino ${ }^{a}$, Università di Torino ${ }^{b}$, Torino, Italy, Università del Piemonte Orientale ${ }^{c}$, Novara, Italy

N. Amapane ${ }^{a, b}$, R. Arcidiacono ${ }^{a, c, 2}, \mathrm{~S}$. Argiro ${ }^{a, b}$, M. Arneodo ${ }^{a, c}$, R. Bellan $^{a, b}$, C. Biino $^{a}$,

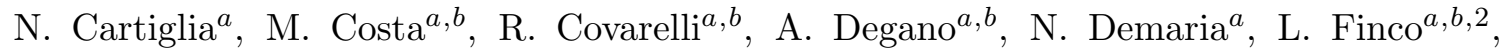
B. Kiani ${ }^{a, b}$, C. Mariotti ${ }^{a}$, S. Maselli ${ }^{a}$, E. Migliore ${ }^{a, b}$, V. Monaco ${ }^{a, b}$, E. Monteil ${ }^{a, b}$, M.M. Obertino ${ }^{a, b}$, L. Pacher ${ }^{a, b}$, N. Pastrone ${ }^{a}$, M. Pelliccioni ${ }^{a}$, G.L. Pinna Angioni ${ }^{a, b}$, F. Ravera ${ }^{a, b}$, A. Romero ${ }^{a, b}$, M. Ruspa ${ }^{a, c}$, R. Sacchi ${ }^{a, b}$, A. Solano ${ }^{a, b}$, A. Staiano ${ }^{a}$, U. Tamponi ${ }^{a}$ 
INFN Sezione di Trieste ${ }^{a}$, Università di Trieste ${ }^{b}$, Trieste, Italy

S. Belforte ${ }^{a}$, V. Candelise ${ }^{a, b, 2}$, M. Casarsa ${ }^{a}$, F. Cossutti ${ }^{a}$, G. Della Ricca ${ }^{a, b}$, B. Gobbo ${ }^{a}$, C. La Licata ${ }^{a, b}$, M. Marone ${ }^{a, b}$, A. Schizzi ${ }^{a, b}$, A. Zanetti ${ }^{a}$

Kangwon National University, Chunchon, Korea

A. Kropivnitskaya, S.K. Nam

Kyungpook National University, Daegu, Korea

D.H. Kim, G.N. Kim, M.S. Kim, D.J. Kong, S. Lee, Y.D. Oh, A. Sakharov, D.C. Son

Chonbuk National University, Jeonju, Korea

J.A. Brochero Cifuentes, H. Kim, T.J. Kim

Chonnam National University, Institute for Universe and Elementary Particles, Kwangju, Korea

S. Song

Korea University, Seoul, Korea

S. Choi, Y. Go, D. Gyun, B. Hong, M. Jo, H. Kim, Y. Kim, B. Lee, K. Lee, K.S. Lee,

S. Lee, S.K. Park, Y. Roh

Seoul National University, Seoul, Korea

H.D. Yoo

University of Seoul, Seoul, Korea

M. Choi, H. Kim, J.H. Kim, J.S.H. Lee, I.C. Park, G. Ryu, M.S. Ryu

Sungkyunkwan University, Suwon, Korea

Y. Choi, J. Goh, D. Kim, E. Kwon, J. Lee, I. Yu

Vilnius University, Vilnius, Lithuania

A. Juodagalvis, J. Vaitkus

National Centre for Particle Physics, Universiti Malaya, Kuala Lumpur, Malaysia

I. Ahmed, A.A. Bin Anuar, Z.A. Ibrahim, J.R. Komaragiri, M.A.B. Md Ali3 ${ }^{33}$, F. Mohamad Idris $^{34}$, W.A.T. Wan Abdullah, M.N. Yusli

Centro de Investigacion y de Estudios Avanzados del IPN, Mexico City, Mexico

E. Casimiro Linares, H. Castilla-Valdez, E. De La Cruz-Burelo, I. Heredia-De La Cruz ${ }^{35}$,

A. Hernandez-Almada, R. Lopez-Fernandez, A. Sanchez-Hernandez

Universidad Iberoamericana, Mexico City, Mexico

S. Carrillo Moreno, F. Vazquez Valencia

Benemerita Universidad Autonoma de Puebla, Puebla, Mexico

I. Pedraza, H.A. Salazar Ibarguen

Universidad Autónoma de San Luis Potosí, San Luis Potosí, Mexico

A. Morelos Pineda 
University of Auckland, Auckland, New Zealand

D. Krofcheck

University of Canterbury, Christchurch, New Zealand

P.H. Butler

National Centre for Physics, Quaid-I-Azam University, Islamabad, Pakistan

A. Ahmad, M. Ahmad, Q. Hassan, H.R. Hoorani, W.A. Khan, T. Khurshid, M. Shoaib

National Centre for Nuclear Research, Swierk, Poland

H. Bialkowska, M. Bluj, B. Boimska, T. Frueboes, M. Górski, M. Kazana, K. Nawrocki,

K. Romanowska-Rybinska, M. Szleper, P. Zalewski

Institute of Experimental Physics, Faculty of Physics, University of Warsaw, Warsaw, Poland

G. Brona, K. Bunkowski, A. Byszuk ${ }^{36}$, K. Doroba, A. Kalinowski, M. Konecki, J. Krolikowski, M. Misiura, M. Olszewski, M. Walczak

Laboratório de Instrumentação e Física Experimental de Partículas, Lisboa, Portugal

P. Bargassa, C. Beirão Da Cruz E Silva, A. Di Francesco, P. Faccioli, P.G. Ferreira Parracho,

M. Gallinaro, N. Leonardo, L. Lloret Iglesias, F. Nguyen, J. Rodrigues Antunes, J. Seixas,

O. Toldaiev, D. Vadruccio, J. Varela, P. Vischia

Joint Institute for Nuclear Research, Dubna, Russia

S. Afanasiev, P. Bunin, M. Gavrilenko, I. Golutvin, I. Gorbunov, A. Kamenev, V. Karjavin,

V. Konoplyanikov, A. Lanev, A. Malakhov, V. Matveev ${ }^{37}$, P. Moisenz, V. Palichik,

V. Perelygin, S. Shmatov, S. Shulha, N. Skatchkov, V. Smirnov, A. Zarubin

Petersburg Nuclear Physics Institute, Gatchina (St. Petersburg), Russia

V. Golovtsov, Y. Ivanov, V. Kim ${ }^{38}$, E. Kuznetsova, P. Levchenko, V. Murzin, V. Oreshkin, I. Smirnov, V. Sulimov, L. Uvarov, S. Vavilov, A. Vorobyev

Institute for Nuclear Research, Moscow, Russia

Yu. Andreev, A. Dermenev, S. Gninenko, N. Golubev, A. Karneyeu, M. Kirsanov, N. Krasnikov, A. Pashenkov, D. Tlisov, A. Toropin

Institute for Theoretical and Experimental Physics, Moscow, Russia

V. Epshteyn, V. Gavrilov, N. Lychkovskaya, V. Popov, I. Pozdnyakov, G. Safronov, A. Spiridonov, E. Vlasov, A. Zhokin

National Research Nuclear University 'Moscow Engineering Physics Institute' (MEPhI), Moscow, Russia

A. Bylinkin

P.N. Lebedev Physical Institute, Moscow, Russia

V. Andreev, M. Azarkin ${ }^{39}$, I. Dremin ${ }^{39}$, M. Kirakosyan, A. Leonidov ${ }^{39}$, G. Mesyats, S.V. Rusakov 
Skobeltsyn Institute of Nuclear Physics, Lomonosov Moscow State University, Moscow, Russia

A. Baskakov, A. Belyaev, E. Boos, V. Bunichev, M. Dubinin ${ }^{40}$, L. Dudko, V. Klyukhin, O. Kodolova, N. Korneeva, I. Lokhtin, I. Myagkov, S. Obraztsov, M. Perfilov, S. Petrushanko, V. Savrin

State Research Center of Russian Federation, Institute for High Energy Physics, Protvino, Russia

I. Azhgirey, I. Bayshev, S. Bitioukov, V. Kachanov, A. Kalinin, D. Konstantinov, V. Krychkine, V. Petrov, R. Ryutin, A. Sobol, L. Tourtchanovitch, S. Troshin, N. Tyurin, A. Uzunian, A. Volkov

University of Belgrade, Faculty of Physics and Vinca Institute of Nuclear Sciences, Belgrade, Serbia

P. Adzic ${ }^{41}$, J. Milosevic, V. Rekovic

Centro de Investigaciones Energéticas Medioambientales y Tecnológicas (CIEMAT), Madrid, Spain

J. Alcaraz Maestre, E. Calvo, M. Cerrada, M. Chamizo Llatas, N. Colino, B. De La Cruz, A. Delgado Peris, D. Domínguez Vázquez, A. Escalante Del Valle, C. Fernandez Bedoya, J.P. Fernández Ramos, J. Flix, M.C. Fouz, P. Garcia-Abia, O. Gonzalez Lopez, S. Goy Lopez, J.M. Hernandez, M.I. Josa, E. Navarro De Martino, A. Pérez-Calero Yzquierdo, J. Puerta Pelayo, A. Quintario Olmeda, I. Redondo, L. Romero, J. Santaolalla, M.S. Soares

Universidad Autónoma de Madrid, Madrid, Spain

C. Albajar, J.F. de Trocóniz, M. Missiroli, D. Moran

Universidad de Oviedo, Oviedo, Spain

J. Cuevas, J. Fernandez Menendez, S. Folgueras, I. Gonzalez Caballero, E. Palencia Cortezon, J.M. Vizan Garcia

Instituto de Física de Cantabria (IFCA), CSIC-Universidad de Cantabria, Santander, Spain

I.J. Cabrillo, A. Calderon, J.R. Castiñeiras De Saa, P. De Castro Manzano, J. Duarte Campderros, M. Fernandez, J. Garcia-Ferrero, G. Gomez, A. Lopez Virto, J. Marco, R. Marco, C. Martinez Rivero, F. Matorras, F.J. Munoz Sanchez, J. Piedra Gomez, T. Rodrigo, A.Y. Rodríguez-Marrero, A. Ruiz-Jimeno, L. Scodellaro, N. Trevisani, I. Vila, R. Vilar Cortabitarte

CERN, European Organization for Nuclear Research, Geneva, Switzerland

D. Abbaneo, E. Auffray, G. Auzinger, M. Bachtis, P. Baillon, A.H. Ball, D. Barney, A. Benaglia, J. Bendavid, L. Benhabib, J.F. Benitez, G.M. Berruti, P. Bloch, A. Bocci, A. Bonato, C. Botta, H. Breuker, T. Camporesi, R. Castello, G. Cerminara, M. D'Alfonso, D. d'Enterria, A. Dabrowski, V. Daponte, A. David, M. De Gruttola, F. De Guio, A. De Roeck, S. De Visscher, E. Di Marco, M. Dobson, M. Dordevic, B. Dorney, T. du Pree, M. Dünser, N. Dupont, A. Elliott-Peisert, G. Franzoni, W. Funk, D. Gigi, K. Gill, D. Giordano, M. Girone, F. Glege, R. Guida, S. Gundacker, M. Guthoff, J. Hammer, 
P. Harris, J. Hegeman, V. Innocente, P. Janot, H. Kirschenmann, M.J. Kortelainen, K. Kousouris, K. Krajczar, P. Lecoq, C. Lourenço, M.T. Lucchini, N. Magini, L. Malgeri, M. Mannelli, A. Martelli, L. Masetti, F. Meijers, S. Mersi, E. Meschi, F. Moortgat, S. Morovic, M. Mulders, M.V. Nemallapudi, H. Neugebauer, S. Orfanelli ${ }^{42}$, L. Orsini, L. Pape, E. Perez, M. Peruzzi, A. Petrilli, G. Petrucciani, A. Pfeiffer, D. Piparo, A. Racz, G. Rolandi ${ }^{43}$, M. Rovere, M. Ruan, H. Sakulin, C. Schäfer, C. Schwick, A. Sharma, P. Silva, M. Simon, P. Sphicas ${ }^{44}$, J. Steggemann, B. Stieger, M. Stoye, Y. Takahashi, D. Treille, A. Triossi, A. Tsirou, G.I. Veres ${ }^{22}$, N. Wardle, H.K. Wöhri, A. Zagozdzinska ${ }^{36}$, W.D. Zeuner

Paul Scherrer Institut, Villigen, Switzerland

W. Bertl, K. Deiters, W. Erdmann, R. Horisberger, Q. Ingram, H.C. Kaestli, D. Kotlinski, U. Langenegger, D. Renker, T. Rohe

Institute for Particle Physics, ETH Zurich, Zurich, Switzerland

F. Bachmair, L. Bäni, L. Bianchini, B. Casal, G. Dissertori, M. Dittmar, M. Donegà, P. Eller, C. Grab, C. Heidegger, D. Hits, J. Hoss, G. Kasieczka, W. Lustermann, B. Mangano, M. Marionneau, P. Martinez Ruiz del Arbol, M. Masciovecchio, D. Meister, F. Micheli, P. Musella, F. Nessi-Tedaldi, F. Pandolfi, J. Pata, F. Pauss, L. Perrozzi, M. Quittnat, M. Rossini, A. Starodumov ${ }^{45}$, M. Takahashi, V.R. Tavolaro, K. Theofilatos, R. Wallny

Universität Zürich, Zurich, Switzerland

T.K. Aarrestad, C. Amsler ${ }^{46}$, L. Caminada, M.F. Canelli, V. Chiochia, A. De Cosa, C. Galloni, A. Hinzmann, T. Hreus, B. Kilminster, C. Lange, J. Ngadiuba, D. Pinna, P. Robmann, F.J. Ronga, D. Salerno, Y. Yang

National Central University, Chung-Li, Taiwan

M. Cardaci, K.H. Chen, T.H. Doan, Sh. Jain, R. Khurana, M. Konyushikhin, C.M. Kuo, W. Lin, Y.J. Lu, S.S. Yu

National Taiwan University (NTU), Taipei, Taiwan

Arun Kumar, R. Bartek, P. Chang, Y.H. Chang, Y.W. Chang, Y. Chao, K.F. Chen, P.H. Chen, C. Dietz, F. Fiori, U. Grundler, W.-S. Hou, Y. Hsiung, Y.F. Liu, R.-S. Lu, M. Miñano Moya, E. Petrakou, J.f. Tsai, Y.M. Tzeng

Chulalongkorn University, Faculty of Science, Department of Physics, Bangkok, Thailand

B. Asavapibhop, K. Kovitanggoon, G. Singh, N. Srimanobhas, N. Suwonjandee

Cukurova University, Adana, Turkey

A. Adiguzel, S. Cerci ${ }^{47}$, Z.S. Demiroglu, C. Dozen, I. Dumanoglu, S. Girgis, G. Gokbulut, Y. Guler, E. Gurpinar, I. Hos, E.E. Kangal ${ }^{48}$, A. Kayis Topaksu, G. Onengut ${ }^{49}$, K. Ozdemir ${ }^{50}$, S. Ozturk ${ }^{51}$, B. Tali ${ }^{47}$, H. Topakli ${ }^{51}$, M. Vergili, C. Zorbilmez

Middle East Technical University, Physics Department, Ankara, Turkey

I.V. Akin, B. Bilin, S. Bilmis, B. Isildak ${ }^{52}$, G. Karapinar ${ }^{53}$, M. Yalvac, M. Zeyrek 
Bogazici University, Istanbul, Turkey

E. Gülmez, M. Kaya ${ }^{54}$, O. Kaya ${ }^{55}$, E.A. Yetkin ${ }^{56}$, T. Yetkin ${ }^{57}$

Istanbul Technical University, Istanbul, Turkey

K. Cankocak, S. Sen ${ }^{58}$, F.I. Vardarl

Institute for Scintillation Materials of National Academy of Science of Ukraine, Kharkov, Ukraine

B. Grynyov

National Scientific Center, Kharkov Institute of Physics and Technology, Kharkov, Ukraine

L. Levchuk, P. Sorokin

\section{University of Bristol, Bristol, United Kingdom}

R. Aggleton, F. Ball, L. Beck, J.J. Brooke, E. Clement, D. Cussans, H. Flacher, J. Goldstein, M. Grimes, G.P. Heath, H.F. Heath, J. Jacob, L. Kreczko, C. Lucas, Z. Meng, D.M. Newbold ${ }^{59}$, S. Paramesvaran, A. Poll, T. Sakuma, S. Seif El Nasr-storey, S. Senkin, D. Smith, V.J. Smith

\section{Rutherford Appleton Laboratory, Didcot, United Kingdom}

K.W. Bell, A. Belyaev ${ }^{60}$, C. Brew, R.M. Brown, L. Calligaris, D. Cieri, D.J.A. Cockerill, J.A. Coughlan, K. Harder, S. Harper, E. Olaiya, D. Petyt, C.H. Shepherd-Themistocleous, A. Thea, I.R. Tomalin, T. Williams, W.J. Womersley, S.D. Worm

\section{Imperial College, London, United Kingdom}

M. Baber, R. Bainbridge, O. Buchmuller, A. Bundock, D. Burton, S. Casasso, M. Citron, D. Colling, L. Corpe, N. Cripps, P. Dauncey, G. Davies, A. De Wit, M. Della Negra, P. Dunne, A. Elwood, W. Ferguson, J. Fulcher, D. Futyan, G. Hall, G. Iles, M. Kenzie, R. Lane, R. Lucas $^{59}$, L. Lyons, A.-M. Magnan, S. Malik, J. Nash, A. Nikitenko ${ }^{45}$, J. Pela, M. Pesaresi, K. Petridis, D.M. Raymond, A. Richards, A. Rose, C. Seez, A. Tapper, K. Uchida, M. Vazquez Acosta ${ }^{61}$, T. Virdee, S.C. Zenz

\section{Brunel University, Uxbridge, United Kingdom}

J.E. Cole, P.R. Hobson, A. Khan, P. Kyberd, D. Leggat, D. Leslie, I.D. Reid, P. Symonds, L. Teodorescu, M. Turner

Baylor University, Waco, U.S.A.

A. Borzou, K. Call, J. Dittmann, K. Hatakeyama, A. Kasmi, H. Liu, N. Pastika

The University of Alabama, Tuscaloosa, U.S.A.

O. Charaf, S.I. Cooper, C. Henderson, P. Rumerio

Boston University, Boston, U.S.A.

A. Avetisyan, T. Bose, C. Fantasia, D. Gastler, P. Lawson, D. Rankin, C. Richardson, J. Rohlf, J. St. John, L. Sulak, D. Zou 
Brown University, Providence, U.S.A.

J. Alimena, E. Berry, S. Bhattacharya, D. Cutts, N. Dhingra, A. Ferapontov, A. Garabedian, J. Hakala, U. Heintz, E. Laird, G. Landsberg, Z. Mao, M. Narain, S. Piperov, S. Sagir, T. Sinthuprasith, R. Syarif

University of California, Davis, Davis, U.S.A.

R. Breedon, G. Breto, M. Calderon De La Barca Sanchez, S. Chauhan, M. Chertok, J. Conway, R. Conway, P.T. Cox, R. Erbacher, M. Gardner, W. Ko, R. Lander, M. Mulhearn, D. Pellett, J. Pilot, F. Ricci-Tam, S. Shalhout, J. Smith, M. Squires, D. Stolp, M. Tripathi, S. Wilbur, R. Yohay

University of California, Los Angeles, U.S.A.

R. Cousins, P. Everaerts, C. Farrell, J. Hauser, M. Ignatenko, D. Saltzberg, E. Takasugi, V. Valuev, M. Weber

University of California, Riverside, Riverside, U.S.A.

K. Burt, R. Clare, J. Ellison, J.W. Gary, G. Hanson, J. Heilman, M. Ivova PANEVA, P. Jandir, E. Kennedy, F. Lacroix, O.R. Long, A. Luthra, M. Malberti, M. Olmedo Negrete, A. Shrinivas, H. Wei, S. Wimpenny, B. R. Yates

University of California, San Diego, La Jolla, U.S.A.

J.G. Branson, G.B. Cerati, S. Cittolin, R.T. D’Agnolo, M. Derdzinski, A. Holzner, R. Kelley, D. Klein, J. Letts, I. Macneill, D. Olivito, S. Padhi, M. Pieri, M. Sani, V. Sharma, S. Simon, M. Tadel, A. Vartak, S. Wasserbaech ${ }^{62}$, C. Welke, F. Würthwein, A. Yagil, G. Zevi Della Porta

University of California, Santa Barbara, Santa Barbara, U.S.A.

D. Barge, J. Bradmiller-Feld, C. Campagnari, A. Dishaw, V. Dutta, K. Flowers, M. Franco Sevilla, P. Geffert, C. George, F. Golf, L. Gouskos, J. Gran, J. Incandela, C. Justus, N. Mccoll, S.D. Mullin, J. Richman, D. Stuart, I. Suarez, W. To, C. West, J. Yoo

California Institute of Technology, Pasadena, U.S.A.

D. Anderson, A. Apresyan, A. Bornheim, J. Bunn, Y. Chen, J. Duarte, A. Mott, H.B. Newman, C. Pena, M. Pierini, M. Spiropulu, J.R. Vlimant, S. Xie, R.Y. Zhu

Carnegie Mellon University, Pittsburgh, U.S.A.

M.B. Andrews, V. Azzolini, A. Calamba, B. Carlson, T. Ferguson, M. Paulini, J. Russ, M. Sun, H. Vogel, I. Vorobiev

University of Colorado Boulder, Boulder, U.S.A.

J.P. Cumalat, W.T. Ford, A. Gaz, F. Jensen, A. Johnson, M. Krohn, T. Mulholland, U. Nauenberg, K. Stenson, S.R. Wagner

Cornell University, Ithaca, U.S.A.

J. Alexander, A. Chatterjee, J. Chaves, J. Chu, S. Dittmer, N. Eggert, N. Mirman, G. Nicolas Kaufman, J.R. Patterson, A. Rinkevicius, A. Ryd, L. Skinnari, L. Soffi, W. Sun, S.M. Tan, W.D. Teo, J. Thom, J. Thompson, J. Tucker, Y. Weng, P. Wittich 
Fermi National Accelerator Laboratory, Batavia, U.S.A.

S. Abdullin, M. Albrow, J. Anderson, G. Apollinari, S. Banerjee, L.A.T. Bauerdick, A. Beretvas, J. Berryhill, P.C. Bhat, G. Bolla, K. Burkett, J.N. Butler, H.W.K. Cheung, F. Chlebana, S. Cihangir, V.D. Elvira, I. Fisk, J. Freeman, E. Gottschalk, L. Gray, D. Green, S. Grünendahl, O. Gutsche, J. Hanlon, D. Hare, R.M. Harris, S. Hasegawa, J. Hirschauer, Z. Hu, S. Jindariani, M. Johnson, U. Joshi, A.W. Jung, B. Klima, B. Kreis, S. Kwan ${ }^{\dagger}$, S. Lammel, J. Linacre, D. Lincoln, R. Lipton, T. Liu, R. Lopes De Sá, J. Lykken, K. Maeshima, J.M. Marraffino, V.I. Martinez Outschoorn, S. Maruyama, D. Mason, P. McBride, P. Merkel, K. Mishra, S. Mrenna, S. Nahn, C. Newman-Holmes, V. O'Dell, K. Pedro, O. Prokofyev, G. Rakness, E. Sexton-Kennedy, A. Soha, W.J. Spalding, L. Spiegel, L. Taylor, S. Tkaczyk, N.V. Tran, L. Uplegger, E.W. Vaandering, C. Vernieri, M. Verzocchi, R. Vidal, H.A. Weber, A. Whitbeck, F. Yang

\section{University of Florida, Gainesville, U.S.A.}

D. Acosta, P. Avery, P. Bortignon, D. Bourilkov, A. Carnes, M. Carver, D. Curry, S. Das, G.P. Di Giovanni, R.D. Field, I.K. Furic, S.V. Gleyzer, J. Hugon, J. Konigsberg, A. Korytov, J.F. Low, P. Ma, K. Matchev, H. Mei, P. Milenovic ${ }^{63}$, G. Mitselmakher, D. Rank, R. Rossin, L. Shchutska, M. Snowball, D. Sperka, N. Terentyev, L. Thomas, J. Wang, S. Wang, J. Yelton

\section{Florida International University, Miami, U.S.A.}

S. Hewamanage, S. Linn, P. Markowitz, G. Martinez, J.L. Rodriguez

Florida State University, Tallahassee, U.S.A.

A. Ackert, J.R. Adams, T. Adams, A. Askew, J. Bochenek, B. Diamond, J. Haas, S. Hagopian, V. Hagopian, K.F. Johnson, A. Khatiwada, H. Prosper, M. Weinberg

Florida Institute of Technology, Melbourne, U.S.A.

M.M. Baarmand, V. Bhopatkar, S. Colafranceschi ${ }^{64}$, M. Hohlmann, H. Kalakhety, D. Noonan, T. Roy, F. Yumiceva

University of Illinois at Chicago (UIC), Chicago, U.S.A.

M.R. Adams, L. Apanasevich, D. Berry, R.R. Betts, I. Bucinskaite, R. Cavanaugh, O. Evdokimov, L. Gauthier, C.E. Gerber, D.J. Hofman, P. Kurt, C. O'Brien, I.D. Sandoval Gonzalez, C. Silkworth, P. Turner, N. Varelas, Z. Wu, M. Zakaria

The University of Iowa, Iowa City, U.S.A.

B. Bilki ${ }^{65}$, W. Clarida, K. Dilsiz, S. Durgut, R.P. Gandrajula, M. Haytmyradov, V. Khristenko, J.-P. Merlo, H. Mermerkaya ${ }^{66}$, A. Mestvirishvili, A. Moeller, J. Nachtman, H. Ogul, Y. Onel, F. Ozok ${ }^{56}$, A. Penzo, C. Snyder, E. Tiras, J. Wetzel, K. Yi

Johns Hopkins University, Baltimore, U.S.A.

I. Anderson, B.A. Barnett, B. Blumenfeld, N. Eminizer, D. Fehling, L. Feng, A.V. Gritsan, P. Maksimovic, C. Martin, M. Osherson, J. Roskes, A. Sady, U. Sarica, M. Swartz, M. Xiao, Y. Xin, C. You 
The University of Kansas, Lawrence, U.S.A.

P. Baringer, A. Bean, G. Benelli, C. Bruner, R.P. Kenny III, D. Majumder, M. Malek, M. Murray, S. Sanders, R. Stringer, Q. Wang

Kansas State University, Manhattan, U.S.A.

A. Ivanov, K. Kaadze, S. Khalil, M. Makouski, Y. Maravin, A. Mohammadi, L.K. Saini, N. Skhirtladze, S. Toda

Lawrence Livermore National Laboratory, Livermore, U.S.A.

D. Lange, F. Rebassoo, D. Wright

University of Maryland, College Park, U.S.A.

C. Anelli, A. Baden, O. Baron, A. Belloni, B. Calvert, S.C. Eno, C. Ferraioli, J.A. Gomez, N.J. Hadley, S. Jabeen, R.G. Kellogg, T. Kolberg, J. Kunkle, Y. Lu, A.C. Mignerey, Y.H. Shin, A. Skuja, M.B. Tonjes, S.C. Tonwar

Massachusetts Institute of Technology, Cambridge, U.S.A.

A. Apyan, R. Barbieri, A. Baty, K. Bierwagen, S. Brandt, W. Busza, I.A. Cali, Z. Demiragli, L. Di Matteo, G. Gomez Ceballos, M. Goncharov, D. Gulhan, Y. Iiyama, G.M. Innocenti, M. Klute, D. Kovalskyi, Y.S. Lai, Y.-J. Lee, A. Levin, P.D. Luckey, A.C. Marini, C. Mcginn, C. Mironov, S. Narayanan, X. Niu, C. Paus, D. Ralph, C. Roland, G. Roland, J. SalfeldNebgen, G.S.F. Stephans, K. Sumorok, M. Varma, D. Velicanu, J. Veverka, J. Wang, T.W. Wang, B. Wyslouch, M. Yang, V. Zhukova

University of Minnesota, Minneapolis, U.S.A.

B. Dahmes, A. Evans, A. Finkel, A. Gude, P. Hansen, S. Kalafut, S.C. Kao, K. Klapoetke, Y. Kubota, Z. Lesko, J. Mans, S. Nourbakhsh, N. Ruckstuhl, R. Rusack, N. Tambe, J. Turkewitz

University of Mississippi, Oxford, U.S.A.

J.G. Acosta, S. Oliveros

University of Nebraska-Lincoln, Lincoln, U.S.A.

E. Avdeeva, K. Bloom, S. Bose, D.R. Claes, A. Dominguez, C. Fangmeier, R. Gonzalez Suarez, R. Kamalieddin, J. Keller, D. Knowlton, I. Kravchenko, J. Lazo-Flores, F. Meier, J. Monroy, F. Ratnikov, J.E. Siado, G.R. Snow

State University of New York at Buffalo, Buffalo, U.S.A.

M. Alyari, J. Dolen, J. George, A. Godshalk, C. Harrington, I. Iashvili, J. Kaisen, A. Kharchilava, A. Kumar, S. Rappoccio, B. Roozbahani

Northeastern University, Boston, U.S.A.

G. Alverson, E. Barberis, D. Baumgartel, M. Chasco, A. Hortiangtham, A. Massironi, D.M. Morse, D. Nash, T. Orimoto, R. Teixeira De Lima, D. Trocino, R.-J. Wang, D. Wood, J. Zhang

Northwestern University, Evanston, U.S.A.

K.A. Hahn, A. Kubik, N. Mucia, N. Odell, B. Pollack, A. Pozdnyakov, M. Schmitt, S. Stoynev, K. Sung, M. Trovato, M. Velasco 
University of Notre Dame, Notre Dame, U.S.A.

A. Brinkerhoff, N. Dev, M. Hildreth, C. Jessop, D.J. Karmgard, N. Kellams, K. Lannon, S. Lynch, N. Marinelli, F. Meng, C. Mueller, Y. Musienko ${ }^{37}$, T. Pearson, M. Planer, A. Reinsvold, R. Ruchti, G. Smith, S. Taroni, N. Valls, M. Wayne, M. Wolf, A. Woodard The Ohio State University, Columbus, U.S.A.

L. Antonelli, J. Brinson, B. Bylsma, L.S. Durkin, S. Flowers, A. Hart, C. Hill, R. Hughes, W. Ji, K. Kotov, T.Y. Ling, B. Liu, W. Luo, D. Puigh, M. Rodenburg, B.L. Winer, H.W. Wulsin

Princeton University, Princeton, U.S.A.

O. Driga, P. Elmer, J. Hardenbrook, P. Hebda, S.A. Koay, P. Lujan, D. Marlow, T. Medvedeva, M. Mooney, J. Olsen, C. Palmer, P. Piroué, X. Quan, H. Saka, D. Stickland, C. Tully, J.S. Werner, A. Zuranski

University of Puerto Rico, Mayaguez, U.S.A.

S. Malik

Purdue University, West Lafayette, U.S.A.

V.E. Barnes, D. Benedetti, D. Bortoletto, L. Gutay, M.K. Jha, M. Jones, K. Jung, D.H. Miller, N. Neumeister, B.C. Radburn-Smith, X. Shi, I. Shipsey, D. Silvers, J. Sun, A. Svyatkovskiy, F. Wang, W. Xie, L. Xu

Purdue University Calumet, Hammond, U.S.A.

N. Parashar, J. Stupak

Rice University, Houston, U.S.A.

A. Adair, B. Akgun, Z. Chen, K.M. Ecklund, F.J.M. Geurts, M. Guilbaud, W. Li, B. Michlin, M. Northup, B.P. Padley, R. Redjimi, J. Roberts, J. Rorie, Z. Tu, J. Zabel

University of Rochester, Rochester, U.S.A.

B. Betchart, A. Bodek, P. de Barbaro, R. Demina, Y. Eshaq, T. Ferbel, M. Galanti, A. Garcia-Bellido, J. Han, A. Harel, O. Hindrichs, A. Khukhunaishvili, G. Petrillo, P. Tan, M. Verzetti

Rutgers, The State University of New Jersey, Piscataway, U.S.A.

S. Arora, A. Barker, J.P. Chou, C. Contreras-Campana, E. Contreras-Campana, D. Duggan, D. Ferencek, Y. Gershtein, R. Gray, E. Halkiadakis, D. Hidas, E. Hughes, S. Kaplan, R. Kunnawalkam Elayavalli, A. Lath, K. Nash, S. Panwalkar, M. Park, S. Salur, S. Schnetzer, D. Sheffield, S. Somalwar, R. Stone, S. Thomas, P. Thomassen, M. Walker

University of Tennessee, Knoxville, U.S.A.

M. Foerster, G. Riley, K. Rose, S. Spanier, A. York

Texas A\&M University, College Station, U.S.A.

O. Bouhali ${ }^{67}$, A. Castaneda Hernandez ${ }^{67}$, M. Dalchenko, M. De Mattia, A. Delgado, S. Dildick, R. Eusebi, J. Gilmore, T. Kamon ${ }^{68}$, V. Krutelyov, R. Mueller, I. Osipenkov, Y. Pakhotin, R. Patel, A. Perloff, A. Rose, A. Safonov, A. Tatarinov, K.A. Ulmer ${ }^{2}$ 


\section{Texas Tech University, Lubbock, U.S.A.}

N. Akchurin, C. Cowden, J. Damgov, C. Dragoiu, P.R. Dudero, J. Faulkner, S. Kunori, K. Lamichhane, S.W. Lee, T. Libeiro, S. Undleeb, I. Volobouev

\section{Vanderbilt University, Nashville, U.S.A.}

E. Appelt, A.G. Delannoy, S. Greene, A. Gurrola, R. Janjam, W. Johns, C. Maguire, Y. Mao, A. Melo, H. Ni, P. Sheldon, B. Snook, S. Tuo, J. Velkovska, Q. Xu

\section{University of Virginia, Charlottesville, U.S.A.}

M.W. Arenton, B. Cox, B. Francis, J. Goodell, R. Hirosky, A. Ledovskoy, H. Li, C. Lin, C. Neu, X. Sun, Y. Wang, E. Wolfe, J. Wood, F. Xia

\section{Wayne State University, Detroit, U.S.A.}

C. Clarke, R. Harr, P.E. Karchin, C. Kottachchi Kankanamge Don, P. Lamichhane, J. Sturdy

\section{University of Wisconsin, Madison, U.S.A.}

D.A. Belknap, D. Carlsmith, M. Cepeda, S. Dasu, L. Dodd, S. Duric, E. Friis, B. Gomber, M. Grothe, R. Hall-Wilton, M. Herndon, A. Hervé, P. Klabbers, A. Lanaro, A. Levine, K. Long, R. Loveless, A. Mohapatra, I. Ojalvo, T. Perry, G.A. Pierro, G. Polese, T. Ruggles, T. Sarangi, A. Savin, A. Sharma, N. Smith, W.H. Smith, D. Taylor, N. Woods

$\dagger$ : Deceased

1: Also at Vienna University of Technology, Vienna, Austria

2: Also at CERN, European Organization for Nuclear Research, Geneva, Switzerland

3: Also at State Key Laboratory of Nuclear Physics and Technology, Peking University, Beijing, China

4: Also at Institut Pluridisciplinaire Hubert Curien, Université de Strasbourg, Université de Haute Alsace Mulhouse, CNRS/IN2P3, Strasbourg, France

5: Also at National Institute of Chemical Physics and Biophysics, Tallinn, Estonia

6: Also at Skobeltsyn Institute of Nuclear Physics, Lomonosov Moscow State University, Moscow, Russia

7: Also at Universidade Estadual de Campinas, Campinas, Brazil

8: Also at Centre National de la Recherche Scientifique (CNRS) - IN2P3, Paris, France

9: Also at Laboratoire Leprince-Ringuet, Ecole Polytechnique, IN2P3-CNRS, Palaiseau, France

10: Also at Joint Institute for Nuclear Research, Dubna, Russia

11: Now at Suez University, Suez, Egypt

12: Also at Beni-Suef University, Bani Sweif, Egypt

13: Now at British University in Egypt, Cairo, Egypt

14: Also at Cairo University, Cairo, Egypt

15: Also at Fayoum University, El-Fayoum, Egypt

16: Also at Université de Haute Alsace, Mulhouse, France

17: Also at Tbilisi State University, Tbilisi, Georgia

18: Also at RWTH Aachen University, III. Physikalisches Institut A, Aachen, Germany

19: Also at University of Hamburg, Hamburg, Germany

20: Also at Brandenburg University of Technology, Cottbus, Germany

21: Also at Institute of Nuclear Research ATOMKI, Debrecen, Hungary 
22: Also at Eötvös Loránd University, Budapest, Hungary

23: Also at University of Debrecen, Debrecen, Hungary

24: Also at Wigner Research Centre for Physics, Budapest, Hungary

25: Also at University of Visva-Bharati, Santiniketan, India

26: Now at King Abdulaziz University, Jeddah, Saudi Arabia

27: Also at University of Ruhuna, Matara, Sri Lanka

28: Also at Isfahan University of Technology, Isfahan, Iran

29: Also at University of Tehran, Department of Engineering Science, Tehran, Iran

30: Also at Plasma Physics Research Center, Science and Research Branch, Islamic Azad University, Tehran, Iran

31: Also at Università degli Studi di Siena, Siena, Italy

32: Also at Purdue University, West Lafayette, U.S.A.

33: Also at International Islamic University of Malaysia, Kuala Lumpur, Malaysia

34: Also at Malaysian Nuclear Agency, MOSTI, Kajang, Malaysia

35: Also at Consejo Nacional de Ciencia y Tecnología, Mexico city, Mexico

36: Also at Warsaw University of Technology, Institute of Electronic Systems, Warsaw, Poland

37: Also at Institute for Nuclear Research, Moscow, Russia

38: Also at St. Petersburg State Polytechnical University, St. Petersburg, Russia

39: Also at National Research Nuclear University 'Moscow Engineering Physics Institute' (MEPhI), Moscow, Russia

40: Also at California Institute of Technology, Pasadena, U.S.A.

41: Also at Faculty of Physics, University of Belgrade, Belgrade, Serbia

42: Also at National Technical University of Athens, Athens, Greece

43: Also at Scuola Normale e Sezione dell'INFN, Pisa, Italy

44: Also at University of Athens, Athens, Greece

45: Also at Institute for Theoretical and Experimental Physics, Moscow, Russia

46: Also at Albert Einstein Center for Fundamental Physics, Bern, Switzerland

47: Also at Adiyaman University, Adiyaman, Turkey

48: Also at Mersin University, Mersin, Turkey

49: Also at Cag University, Mersin, Turkey

50: Also at Piri Reis University, Istanbul, Turkey

51: Also at Gaziosmanpasa University, Tokat, Turkey

52: Also at Ozyegin University, Istanbul, Turkey

53: Also at Izmir Institute of Technology, Izmir, Turkey

54: Also at Marmara University, Istanbul, Turkey

55: Also at Kafkas University, Kars, Turkey

56: Also at Mimar Sinan University, Istanbul, Istanbul, Turkey

57: Also at Yildiz Technical University, Istanbul, Turkey

58: Also at Hacettepe University, Ankara, Turkey

59: Also at Rutherford Appleton Laboratory, Didcot, United Kingdom

60: Also at School of Physics and Astronomy, University of Southampton, Southampton, United Kingdom

61: Also at Instituto de Astrofísica de Canarias, La Laguna, Spain

62: Also at Utah Valley University, Orem, U.S.A.

63: Also at University of Belgrade, Faculty of Physics and Vinca Institute of Nuclear Sciences, Belgrade, Serbia

64: Also at Facoltà Ingegneria, Università di Roma, Roma, Italy

65: Also at Argonne National Laboratory, Argonne, U.S.A. 
66: Also at Erzincan University, Erzincan, Turkey

67: Also at Texas A\&M University at Qatar, Doha, Qatar

68: Also at Kyungpook National University, Daegu, Korea 